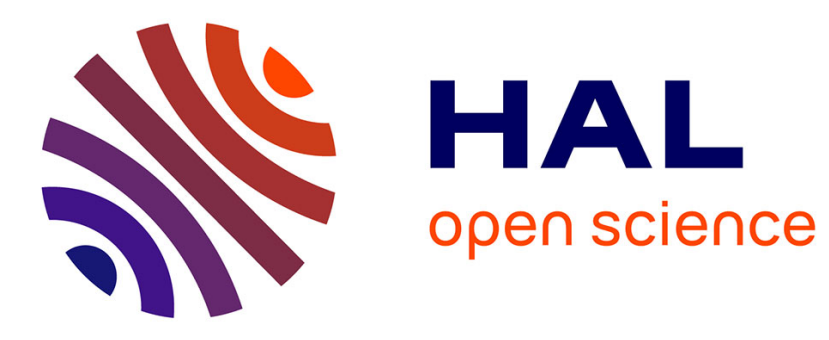

\title{
Mellin analysis of weighted Sobolev spaces with nonhomogeneous norms on cones
}

Martin Costabel, Monique Dauge, Serge Nicaise

\section{To cite this version:}

Martin Costabel, Monique Dauge, Serge Nicaise. Mellin analysis of weighted Sobolev spaces with nonhomogeneous norms on cones. Ari Laptev, Tamara Rozhkovskaya. Around the Research of Vladimir Maz'ya I, Function Spaces., Springer, pp.105-136, 2010, International Mathematical Series volume 11. hal-00402645

\section{HAL Id: hal-00402645 \\ https://hal.science/hal-00402645}

Submitted on 7 Jul 2009

HAL is a multi-disciplinary open access archive for the deposit and dissemination of scientific research documents, whether they are published or not. The documents may come from teaching and research institutions in France or abroad, or from public or private research centers.
L'archive ouverte pluridisciplinaire HAL, est destinée au dépôt et à la diffusion de documents scientifiques de niveau recherche, publiés ou non, émanant des établissements d'enseignement et de recherche français ou étrangers, des laboratoires publics ou privés. 


\title{
MELLIN ANALYSIS OF WEIGHTED SOBOLEV SPACES WITH NONHOMOGENEOUS NORMS ON CONES
}

\author{
MARTIN COSTABEL, MONIQUE DAUGE, AND SERGE NICAISE
}

\begin{abstract}
On domains with conical points, weighted Sobolev spaces with powers of the distance to the conical points as weights form a classical framework for describing the regularity of solutions of elliptic boundary value problems, cf. papers by Kondrat'ev and Maz'ya-Plamenevskii. Two classes of weighted norms are usually considered: Homogeneous norms, where the weight exponent varies with the order of derivatives, and nonhomogeneous norms, where the same weight is used for all orders of derivatives. For the analysis of the spaces with homogeneous norms, Mellin transformation is a classical tool. In this paper, we show how Mellin transformation can also be used to give an optimal characterization of the structure of weighted Sobolev spaces with nonhomogeneous norms on finite cones in the case of both non-critical and critical indices. This characterization can serve as a basis for the proof of regularity and Fredholm theorems in such weighted Sobolev spaces on domains with conical points, even in the case of critical indices.
\end{abstract}

\section{CONTENTS}

1. Introduction 1

2. Notation: Weighted Sobolev spaces on cones 3

3. Characterizations by Mellin transformation techniques 6

4. Structure of spaces with nonhomogeneous norms in the critical case 21

5. Conclusion 27

$\begin{array}{ll}\text { Addresses } & 28\end{array}$

\section{INTRODUCTION}

When analyzing elliptic regularity in the neighborhood of a conical point on the boundary of an otherwise smooth domain, one is faced with a dilemma:

Near the singular point, the conical geometry suggests the use of estimates in weighted Sobolev spaces with homogeneous norms, and a well-known tool for analyzing them and for obtaining the estimates is the Mellin transformation. This analysis is carried out in the classical paper [3] by Kondrat'ev.

On the other hand, since this analysis corresponds to a blow-up of the corner, that is a diffeomorphism between the tangent cone and an infinite cylinder, the conical point moves to infinity, and therefore functions in this class of spaces always have trivial Taylor expansions at the corner. Depending on the weight index, they either have no controlled behavior at the corner at all or they tend to zero. If one wants to study inhomogeneous boundary 
value problems, then smooth right hand sides and the corresponding solutions will require spaces that allow the description of non-trivial Taylor expansions at corner points.

Appropriate spaces have been analyzed using tools from real analysis by Maz'ya and Plamenevskii [6]. Such spaces can be defined by nonhomogeneous weighted norms, where the weight exponent is the same for all derivatives. The simplest examples are ordinary, non-weighted Sobolev norms. As presented in detail in the book [4] by Kozlov, Maz'ya and Rossmann, the analysis of these spaces with nonhomogeneous norms shows several peculiarities:

1. For a given space dimension $n$ and Sobolev order $m$, there is a finite set of exceptional, "critical" weight exponents $\beta$, characterized in our notation by the condition

$$
-\beta-\frac{n}{2}=\eta \in \mathbb{N} ; \quad 0 \leqslant \eta \leqslant m-1
$$

such that for the non-critical case, the space with nonhomogeneous norm splits into the direct sum of a space with homogeneous norm and a space of polynomials, corresponding to the Taylor expansion at the corner. In the critical case, the splitting involves an infinitedimensional space of generalized polynomials. The study of the critical cases is of practical importance, because for example in two-dimensional domains, the ordinary Sobolev spaces with integer order are all in the critical case $\eta=m-1$.

2. The relation of the spaces with nonhomogeneous norms with respect to Taylor expansions at the corner is somewhat complicated, depending on the weight and the order: For $\eta<0$, the space with nonhomogeneous norm coincides with the corresponding space with homogeneous norm and contains all polynomials, but has no controlled Taylor expansion. For $0 \leqslant \eta<m$, the nonhomogeneous norm still allows all polynomials and controls the Taylor expansion of order $[\eta]$ at the corner. If $\eta \geqslant m$, then the space with nonhomogeneous norm again coincides with the corresponding space with homogeneous norm and has vanishing Taylor expansion of order $m-1$. Thus there are two (non-disjoint) classes of spaces involved, and the weighted Sobolev spaces with nonhomogeneous norms fall into one or the other of these classes, namely the class of spaces with homogeneous norms on one hand and a class of spaces with weighted norms and nontrivial Taylor expansion on the other hand.

3. Whereas the definition of the non-homogeneous norms is simple, it turns out that for the analysis of the spaces one also needs descriptions by more complicated equivalent norms, where the weight exponent does depend, in a specific way, on the order of the derivatives. Such "step-weighted" Sobolev spaces have been studied by Nazarov [7, 8].

In [4], the analysis of the weighted Sobolev spaces with nonhomogeneous norms is presented using real-variable tools, in particular techniques based on Hardy's inequality.

In this paper, we present an analysis of the spaces with nonhomogeneous norms based on Mellin transformation. We show how the three points described above can be achieved in an optimal way. In particular,

1. we characterize the spaces with nonhomogeneous norms via Mellin transformation in the non-critical and in the critical case;

2. we give a natural definition via Mellin transforms of the second class of spaces mentioned in point 2 above, namely the spaces with weighted norms and nontrivial Taylor expansions; 
MELLIN ANALYSIS OF WEIGHTED SOBOLEV SPACES WITH NONHOMOGENEOUS NORMS ON CONES3

3. we show how the question of equivalent norms can be solved via Mellin transformation.

The analysis in this paper is a generalization of the Mellin characterization of standard Sobolev spaces that was introduced in [2] for the analysis of elliptic regularity on domains with corners. For the case of critical weight exponents, we give a Mellin description of the generalized Taylor expansion that was introduced and analyzed with real-variable techniques in [4]. Based on our Mellin characterization, one can obtain Fredholm theorems and elliptic regularity results, in particular analytic regularity results, on domains with conical points. This is developed in the forthcoming work [1].

\section{Notation: Weighted Sobolev SPACES on CONES}

A regular cone $K \subset \mathbb{R}^{n}, n \geqslant 2$ is an unbounded open set of the form

$$
K=\left\{\mathbf{x} \in \mathbb{R}^{n} \backslash\{\mathbf{0}\}: \frac{\mathbf{x}}{|\mathbf{x}|} \in G\right\},
$$

where $G$ is a smooth domain of the unit sphere $\mathbb{S}^{n-1}$ called the solid angle of $K$. Note that if $n=2$, this implies that $K$ has a Lipschitz boundary (excluding domains with cracks), which is not necessarily the case if $n \geqslant 3$. Note further that our analysis below is also valid in the case of domains with cracks.

The finite cone $S$ associated with $K$ is simply

$$
S=K \cap B(\mathbf{0}, 1) .
$$

In the one-dimensional case, we consider $K=\mathbb{R}_{+}$and $S=(0,1)$, which corresponds to $G=\{1\}$.

For $k \in \mathbb{N},\|\cdot\|_{k ; O}$ denotes the standard Sobolev norm of $H^{k}(O)$.

2.1. Weighted spaces with homogeneous norms. The spaces on which relies a large part of our analysis are the "classical" weighted spaces of Kondrat'ev. The "originality" of our definition is a new convention for their notation.

Definition 2.1. $\quad \star$ Let $\beta$ be a real number and let $m \geqslant 0$ be an integer.

$\star \quad \beta$ is called the weight exponent and $m$ the Sobolev exponent.

* The weighted space with homogeneous norm $\mathrm{K}_{\beta}^{m}(K)$ is defined by

$$
\mathrm{K}_{\beta}^{m}(K)=\left\{u \in \mathrm{L}_{\text {loc }}^{2}(K): r^{\beta+|\alpha|} \partial_{\mathbf{x}}^{\alpha} u \in \mathrm{L}^{2}(K), \quad \forall \alpha,|\alpha| \leqslant m\right\}
$$

and endowed with semi-norm and norm respectively defined as

$$
|u|_{\mathrm{K}_{\beta}^{m}(K)}^{2}=\sum_{|\alpha|=m}\left\|r^{\beta+|\alpha|} \partial_{\mathbf{x}}^{\alpha} u\right\|_{0 ; K}^{2} \text { and }\|u\|_{\mathrm{K}_{\beta}^{m}(K)}^{2}=\sum_{k=0}^{m}|u|_{\mathrm{K}_{\beta}^{k}(K)}^{2} .
$$

The weighted spaces introduced by Kondrat'ev in [3] are denoted by $\stackrel{\circ}{W}_{\alpha}^{m}(K)$. The correspondence with our notation is

$$
\stackrel{\circ}{W}_{\alpha}^{m}(K)=\mathrm{K}_{\frac{\alpha}{2}-m}^{m}(K) \text { i.e. } \quad \mathrm{K}_{\beta}^{m}(K)=\stackrel{0}{W_{2 \beta+2 m}^{m}}(K) .
$$


These spaces are also of constant use in related works by Kozlov, Maz'ya, Nazarov, Plamenevskii, Rossmann, see the monographs $[9,4,5]$ for example. They are denoted by $V_{\beta}^{m}(K)$ with the following correspondence with our spaces

$$
V_{\beta}^{m}(K)=\mathrm{K}_{\beta-m}^{m}(K) \text { i.e. } \mathrm{K}_{\beta}^{m}(K)=V_{\beta+m}^{m}(K) .
$$

We choose the convention in (2.3) because it simplifies some statements: An obvious, but fundamental property of the scale $\mathrm{K}_{\beta}$ is its monotonicity with respect to $m$

$$
\mathrm{K}_{\beta}^{m+1}(K) \subset \mathrm{K}_{\beta}^{m}(K), \quad m \in \mathbb{N} .
$$

This allows a simple definition of $\mathscr{C}^{\infty}$ and analytic functions with weight, see Definition 4.1. Also, in mapping properties of differential operators with constant coefficients, as well as in elliptic regularity theorems ("shift theorem"), the shift in the weight exponent $\beta$ is independent of the regularity parameter $m$, in contrast to what happens with the Kondrat'ev or the $V_{\beta}^{m}$ spaces.

The space $\mathrm{K}_{\beta}^{m}(S)$ with its semi-norm $|\cdot| \mathrm{K}_{\beta}^{m}(S)$ and norm $\|\cdot\|_{\mathrm{K}_{\beta}^{m}(S)}$ is defined similarly by replacing $K$ by $S$.

\subsection{Weighted spaces with nonhomogeneous norms.}

Definition 2.2. $\quad \star$ Let $\beta$ be a real number and $m \geqslant 0$ be an integer.

* The weighted space with non-homogeneous norm $\mathrm{J}_{\beta}^{m}(S)$ is defined by

$$
\mathrm{J}_{\beta}^{m}(S)=\left\{u \in \mathrm{L}_{\text {loc }}^{2}(S): r^{\beta+m} \partial_{\mathbf{x}}^{\alpha} u \in \mathrm{L}^{2}(S), \quad \forall \alpha,|\alpha| \leqslant m\right\}
$$

with its norm

$$
\|u\|_{J_{\beta}^{m}(S)}^{2}=\sum_{|\alpha| \leqslant m}\left\|r^{\beta+m} \partial_{\mathbf{x}}^{\alpha} u\right\|_{0 ; S}^{2} .
$$

The semi-norm of $\mathrm{J}_{\beta}^{m}(S)$ coincides with the semi-norm of $\mathrm{K}_{\beta}^{m}(S)$ :

$$
|u|_{J_{\beta}^{m}(S)}^{2}=|u|_{\mathrm{K}_{\beta}^{m}(S)}^{2}=\sum_{|\alpha|=m}\left\|r^{\beta+|\alpha|} \partial_{\mathbf{x}}^{\alpha} u\right\|_{0 ; S}^{2} .
$$

* The space $\mathrm{J}_{\beta}^{m}(K)$ with its norm and semi-norm is defined in the same way.

Our space $\mathrm{J}_{\beta}^{m}(S)$ is the same as the space denoted by $W_{2, \beta+m}^{m}(S)$ in [4].

The following properties are obvious consequences of the definitions:

Lemma 2.3. $\quad$ a) For all $\beta<\beta^{\prime}$ we have the embedding $\mathrm{J}_{\beta}^{m}(S) \subset \mathrm{J}_{\beta^{\prime}}^{m}(S)$.

b) We have the embeddings for all $\beta \in \mathbb{R}$ and $m \in \mathbb{N}$

$$
\mathrm{K}_{\beta}^{m}(S) \subset \mathrm{J}_{\beta}^{m}(S) \subset \mathrm{K}_{\beta+m}^{m}(S) .
$$

c) Let $\alpha \in \mathbb{N}^{n}$ be a multi-index of length $|\alpha|=k \leqslant m$. Then the partial differential operator $\partial_{\mathbf{x}}^{\alpha}$ is continuous from $\mathrm{J}_{\beta}^{m}(S)$ into $\mathrm{J}_{\beta+k}^{m-k}(S)$.

In contrast to the scale $\mathrm{K}_{\beta}^{m}$, we do not necessarily have the inclusion of the space $\mathrm{J}_{\beta}^{m}(S)$ in $\mathrm{J}_{\beta}^{m-1}(S)$. We will see (Corollary 3.19) that such an inclusion does hold when $m$ is large enough, which allows the definition of $\mathrm{J}_{\beta}^{\infty}(S)$ and of the corresponding analytic class.

A remarkable and unusual property of the spaces $\mathrm{J}_{\beta}^{m}(S)$ is that we do not, in general, obtain an equivalent norm for $\mathrm{J}_{\beta}^{m}(S)$ if we retain in (2.5) only the semi-norm $(|\alpha|=m)$ 
MELLIN ANALYSIS OF WEIGHTED SOBOLEV SPACES WITH NONHOMOGENEOUS NORMS ON CONES5

and the $\mathrm{L}^{2}$ norm $(|\alpha|=0)$. A counter-example for such an equivalence is obtained with the following choice

$$
m \geqslant 2, \quad m<\eta=-\beta-\frac{n}{2}<m+1, \quad u=x_{1} .
$$

Then the function $r^{\beta+m} \partial_{\mathbf{x}}^{\alpha} u$ is square integrable for $|\alpha|=0$ and for $|\alpha| \geqslant 2$, but not for $\alpha=(1,0, \ldots, 0)$. See $\S 3.4$ for further details.

We shall need more precise comparisons between the $\mathrm{K}$ and $\mathrm{J}$ spaces than the embeddings (2.7). As we will show later on, the space $\mathrm{K}_{\beta}^{m}(S)$ may be closed with finite codimension in $\mathrm{J}_{\beta}^{m}(S)$ (non-critical case), or not closed with infinite codimension (critical case). In the following lemma we compare the properties of inclusion of the space $\mathscr{C}^{\infty}(\bar{S})$ of smooth functions:

Lemma 2.4. Let $\beta \in \mathbb{R}$ and $m \in \mathbb{N}$. Let $\eta=-\beta-\frac{n}{2}$.

a) The space $\mathscr{C}^{\infty}(\bar{S})$ is embedded in $\mathrm{K}_{\beta}^{m}(S)$ if and only if $\eta<0$.

b) The space $\mathscr{C}^{\infty}(\bar{S})$ is embedded in $\mathrm{J}_{\beta}^{m}(S)$ if and only if $\eta<m$.

Proof. Using polar coordinates and the Cauchy-Schwarz inequality, we see that

$$
\mathrm{L}^{\infty}(S) \subset \mathrm{L}_{\beta}^{2}(S) \Longleftrightarrow \beta>-\frac{n}{2} .
$$

The sufficiency follows by using this for all derivatives of $u \in \mathscr{C}^{\infty}(\bar{S})$.

We find the necessity of the conditions on $\eta$ by considering the constant function $u=1$ in both cases.

Concerning spaces of finite regularity, it follows from the definition that the standard Sobolev space $\mathrm{H}^{m}$ without weight coincides with $\mathrm{J}_{-m}^{m}$. For the Sobolev spaces $\mathrm{H}^{m}$ we have the embeddings corresponding to (2.7), namely

$$
\mathrm{K}_{-m}^{m}(S) \subset \mathrm{H}^{m}(S) \subset \mathrm{K}_{0}^{m}(S) .
$$

In addition we know from the Sobolev embedding theorem that if $k$ is a non-negative integer such that $k<m-\frac{n}{2}$, we have the embeddings

$$
\mathrm{H}^{m}(S) \subset \mathscr{C}^{k}(\bar{S}) \subset \mathrm{H}^{k}(S) \text {. }
$$

In particular, for elements of $\mathrm{H}^{m}(S)$ all derivatives of length $|\alpha| \leqslant k$ have a trace at the vertex 0 . On the other hand, by density of smooth functions which are zero at the vertex, the elements of $\mathrm{K}_{\beta}^{m}(S)$, as soon as they have traces, have zero traces at the vertex.

One can expect that the spaces $J$ have vertex traces similar to the standard Sobolev spaces. The investigation of this question will be the key to the comparison between the $\mathrm{J}$ spaces and the $\mathrm{K}$ spaces.

Using the same simple argument as in the proof of Lemma 2.4, we find the conditions for the inclusion of polynomials in the weighted Sobolev spaces.

We denote by $\mathbb{P}^{M}(S)$ the space of polynomial functions of degree $\leqslant M$ on $S$ and by $\mathrm{P}^{M}(S)$ the space of homogeneous polynomials of degree $M$.

Lemma 2.5. Let $\beta \in \mathbb{R}$ and $m, k \in \mathbb{N}$. Let $\eta=-\beta-\frac{n}{2}$.

a) $\mathbb{P}^{k}(S) \subset \mathrm{K}_{\beta}^{m}(S) \Longleftrightarrow \mathbb{P}^{0}(S) \subset \mathrm{K}_{\beta}^{m}(S) \Longleftrightarrow \eta<0$.

b) $\mathbb{P}^{k}(S) \subset J_{\beta}^{m}(S) \quad \Longleftrightarrow \mathbb{P}^{0}(S) \subset J_{\beta}^{m}(S) \quad \Longleftrightarrow \eta<m$. 
This complete similarity between the $\mathrm{K}$ spaces and the $\mathrm{J}$ spaces is no longer present if we refine the probe by considering the space of homogeneous polynomials. Still using the same simple argument based on finiteness of norms, we now get

Lemma 2.6. Let $\beta \in \mathbb{R}$ and $m, k \in \mathbb{N}$. Let $\eta=-\beta-\frac{n}{2}$.
a) $\mathrm{P}^{k}(S) \subset \mathrm{K}_{\beta}^{m}(S) \Longleftrightarrow \eta<k$.
b) If $k \geqslant m$, then $\quad \mathrm{P}^{k}(S) \subset \mathrm{J}_{\beta}^{m}(S) \Longleftrightarrow \eta<k$.
c) If $k \leqslant m-1$, then $\quad \mathrm{P}^{k}(S) \subset \mathrm{J}_{\beta}^{m}(S) \Longleftrightarrow \mathrm{P}^{0}(S) \subset \mathrm{J}_{\beta}^{m}(S) \Longleftrightarrow \eta<m$.

As we will show in the following, the question of inclusion of polynomials completely characterizes the structure of the spaces $\mathrm{J}_{\beta}^{m}(S)$ and their corner behavior.

\section{Characterizations by Mellin transformation techniques}

The homogeneous weighted Sobolev norms can be expressed by Mellin transformation, which is the Fourier transformation associated with the group of dilations. We first recall this characterization from Kondrat'ev's classical work [3]. Then we generalize it to include non-homogeneous weighted Sobolev norms, based on the observation that the non-homogeneous norms are defined by sums of homogeneous semi-norms.

3.1. Mellin characterization of spaces with homogeneous norms. In this section, we recall the basic results from [3].

For a function $u$ in $\mathscr{C}_{0}^{\infty}((0, \infty))$ the Mellin transform $\mathscr{M}[u]$ is defined for any complex number $\lambda$ by the integral

$$
\mathscr{M}[u](\lambda)=\int_{0}^{\infty} r^{-\lambda} u(r) \frac{\mathrm{d} r}{r} .
$$

The function $\lambda \mapsto \mathscr{M}[u](\lambda)$ is then holomorphic on the entire complex plane $\mathbb{C}$. Note that $\mathscr{M}[u](\lambda)$ coincides with the Fourier-Laplace transform at $i \lambda$ of the function $t \mapsto u\left(\mathrm{e}^{t}\right)$.

Now any function $u$ defined on our cone $K$ can be naturally written in polar coordinates as

$$
\mathbb{R}_{+} \times G \ni(r, \vartheta) \longmapsto u(\mathbf{x})=u(r \vartheta)
$$

If $u$ has a compact support which does not contain the vertex $\mathbf{0}$, the Mellin transform of $u$ at $\lambda \in \mathbb{C}$ is the function $\mathscr{M}[u](\lambda): \vartheta \mapsto \mathscr{M}[u](\lambda, \vartheta)$ defined on $G$ by

$$
\mathscr{M}[u](\lambda, \vartheta)=\int_{0}^{\infty} r^{-\lambda} u(r \vartheta) \frac{\mathrm{d} r}{r}, \quad \vartheta \in G .
$$

If we define the function $\widetilde{u}$ on the cylinder $\mathbb{R} \times G$ by $\widetilde{u}(t, \vartheta)=u\left(\mathrm{e}^{t} \vartheta\right)$, we see that the Mellin transform of $u$ at $\lambda$ is the partial Fourier-Laplace transform of $\widetilde{u}$ at $-i \lambda$.

Hence the Mellin transform of a function $u \in \mathscr{C}_{0}^{\infty}(K)$ is holomorphic with values in $\mathscr{C}_{0}^{\infty}(G)$. On the other hand, if $u$ is simply in $\mathrm{L}^{2}(K)$, the function $\mathrm{e}^{\frac{n}{2} t} \widetilde{u}$ belongs to $\mathrm{L}^{2}$ on the cylinder $\mathbb{R} \times G$ and $\lambda \mapsto \mathscr{M}[u](\lambda)$ therefore defines an $\mathrm{L}^{2}$ function on the line $\operatorname{Re} \lambda=-\frac{n}{2}$, with values in $\mathrm{L}^{2}(G)$.

More generally, the Mellin transformation extends to functions $u$ given in a weighted space $\mathrm{K}_{\beta}^{0}(K)$ with a fixed real number $\beta$ : Since $r^{\beta} u$ belongs to $\mathrm{L}^{2}(K)$, the function $\widetilde{u}$ in turn satisfies that $\mathrm{e}^{\left(\beta+\frac{n}{2}\right) t} \widetilde{u}$ belongs to $\mathrm{L}^{2}(\mathbb{R} \times G)$. Therefore $\lambda \mapsto \mathscr{M}[u](\lambda)$ defines an $\mathrm{L}^{2}$ 
function on the line $\operatorname{Re} \lambda=-\beta-\frac{n}{2}$. If $u$ belongs to $\mathrm{K}_{\beta}^{m}(K)$, then there appear parameterdependent $\mathrm{H}^{m}$ norms for its Mellin transform, which motivates the following definition.

Definition 3.1. Let $G$ be the solid angle of a regular cone $K$. Let $m \in \mathbb{N}$.

$\star$ For $\lambda \in \mathbb{C}$, the parameter-dependent $\mathrm{H}^{m}$ norm on $G$ is defined by

$$
\|U\|_{m ; G ; \lambda}^{2}=\sum_{k=0}^{m}|\lambda|^{2 m-2 k}\|U\|_{k ; G}^{2} .
$$

* Let $\lambda \mapsto U(\lambda)$ be a function with values in $\mathrm{H}^{m}(G)$, defined for $\lambda$ in a strip $b_{0}<$ $\operatorname{Re} \lambda<b_{1}$. Then for any $b \in\left(b_{0}, b_{1}\right)$, we set

$$
\mathcal{N}_{G}^{m}(U, b)=\left\{\int_{\operatorname{Re} \lambda=b}\|U(\lambda)\|_{m ; G ; \lambda}^{2} \mathrm{~d} \operatorname{Im} \lambda\right\}^{\frac{1}{2}},
$$

and

$$
\mathcal{N}_{G}^{m}\left(U,\left[b_{0}, b_{1}\right]\right)=\sup _{b \in\left(b_{0}, b_{1}\right)} \mathcal{N}_{G}^{m}(U, b)
$$

Later on, we will use the following observation: Let $\lambda \mapsto U(\lambda)$ be meromorphic for $b_{0}<\operatorname{Re} \lambda<b_{1}$ with values in $\mathrm{H}^{m}(G)$. If $\mathcal{N}_{G}^{m}\left(U,\left[b_{0}, b_{1}\right]\right)$ is finite, then $U$ is actually holomorphic. In fact, if $U$ has a pole in $\lambda_{0}$, then $\mathcal{N}_{G}^{m}(U, b)$ is bounded from below by $C\left|b-\operatorname{Re} \lambda_{0}\right|^{-1}$.

As a consequence of the isomorphism between $\mathrm{K}_{\beta}^{m}(K)$ and $\mathrm{H}_{\beta+\frac{n}{2}}^{m}(\mathbb{R} \times G)$, one gets:

Theorem 3.2. Let $\beta$ be a real number and $m \in \mathbb{N}$. Let

$$
\eta:=-\beta-\frac{n}{2} \quad \text { and } \quad \mathfrak{R}[\eta]:=\{\lambda \in \mathbb{C}: \operatorname{Re} \lambda=\eta\} .
$$

The Mellin transformation (3.2) $u \mapsto \mathscr{M}[u]$ induces an isomorphism from $\mathrm{K}_{\beta}^{m}(K)$ onto the space of functions $U: \mathfrak{R}[\eta] \times G \ni(\lambda, \vartheta) \mapsto U(\lambda, \vartheta)$ with finite norm $\mathcal{N}_{G}^{m}(U, \eta)$. The inverse Mellin transform can be written as:

$$
u(\mathbf{x})=\frac{1}{2 i \pi} \int_{\operatorname{Re} \lambda=\eta} r^{\lambda} \mathscr{M}[u](\lambda)(\vartheta) \mathrm{d} \lambda, \quad \mathbf{x}=r \vartheta .
$$

From this Theorem, we see immediately that if $u$ belongs to the intersection of two weighted spaces $\mathrm{K}_{\beta}^{m}(K)$ and $\mathrm{K}_{\beta^{\prime}}^{m}(K)$ with $\beta<\beta^{\prime}$, the Mellin transform of $u$ is defined on two different lines in $\mathbb{C}$. Since $u$ belongs also to all intermediate spaces $\mathrm{K}_{\beta^{\prime \prime}}^{m}(K)$ for $\beta \leqslant$ $\beta^{\prime \prime} \leqslant \beta^{\prime}$, the Mellin transform is defined in a complex strip. In fact, the Mellin transform of $u$ is holomorphic in this strip, and this characterizes the intersection of weighted spaces with different weights, as stated in the following theorem.

Theorem 3.3. Let $\beta<\beta^{\prime}$ two real numbers and $m \in \mathbb{N}$. Let

$$
\eta:=-\beta-\frac{n}{2} \quad \text { and } \quad \eta^{\prime}:=-\beta^{\prime}-\frac{n}{2} .
$$

a) Let $u \in \mathrm{K}_{\beta}^{m}(K) \cap \mathrm{K}_{\beta^{\prime}}^{m}(K)$. Then the Mellin transform $U:=\mathscr{M}[u]$ of $u$ is holomorphic in the open strip $\eta^{\prime}<\operatorname{Re} \lambda<\eta$ with values in $\mathrm{H}^{m}(G)$ and satisfies the following boundedness condition:

$$
\mathcal{N}_{G}^{m}\left(U,\left[\eta^{\prime}, \eta\right]\right) \leqslant C\left(\|u\|_{\mathrm{K}_{\beta}^{m}(K)}+\|u\|_{\mathrm{K}_{\beta^{\prime}}^{m}(K)}\right) .
$$


b) Let $U$ be a holomorphic function in the open strip $\eta^{\prime}<\operatorname{Re} \lambda<\eta$ with values in $\mathrm{H}^{m}(G)$, satisfying $\mathcal{N}_{G}^{m}\left(U,\left[\eta^{\prime}, \eta\right]\right)<\infty$. Then the mapping

$$
b \longmapsto((\xi, \vartheta) \longmapsto U(b+i \xi, \vartheta))
$$

has limits as $b \rightarrow \eta$ and $b \rightarrow \eta^{\prime}$, and the inverse Mellin transforms

$$
u^{\prime}=\frac{1}{2 i \pi} \int_{\operatorname{Re} \lambda=\eta^{\prime}} r^{\lambda} U(\lambda) \mathrm{d} \lambda \text { and } u=\frac{1}{2 i \pi} \int_{\operatorname{Re} \lambda=\eta} r^{\lambda} U(\lambda) \mathrm{d} \lambda,
$$

coincide with each other and define an element of $\mathrm{K}_{\beta}^{m}(K) \cap \mathrm{K}_{\beta^{\prime}}^{m}(K)$.

In the following theorem, we recall the close relation between asymptotic expansions and meromorphic Mellin transforms.

Theorem 3.4. Let $\beta<\beta^{\prime}$ be two real numbers and set

$$
\eta=-\beta-\frac{n}{2} \quad \text { and } \quad \eta^{\prime}=-\beta^{\prime}-\frac{n}{2} .
$$

Let $\lambda_{0}$ be a complex number such that $\eta^{\prime}<\operatorname{Re} \lambda_{0}<\eta$. Let $q$ be a non-negative integer and $\varphi_{0}, \ldots, \varphi_{q}$ be fixed elements of $\mathrm{L}^{2}(G)$.

a) Let $u^{\prime} \in \mathrm{K}_{\beta^{\prime}}^{0}(K)$ such that the identity

$$
u(\mathbf{x})=u^{\prime}(\mathbf{x})+r^{\lambda_{0}} \sum_{j=0}^{q} \frac{1}{j !} \log ^{j} r \varphi_{j}(\vartheta)
$$

defines a function $u$ in $\mathrm{K}_{\beta}^{0}(K)$. Then the Mellin transform $U$ of $u^{\prime}$, defined for $\operatorname{Re} \lambda=\eta^{\prime}$, has a meromorphic extension to the strip $\eta^{\prime}<\operatorname{Re} \lambda<\eta$ such that the function $V$ defined as

$$
V(\lambda):=U(\lambda)-\sum_{j=0}^{q} \frac{\varphi_{j}}{\left(\lambda-\lambda_{0}\right)^{j+1}}
$$

is holomorphic in $\eta^{\prime}<\operatorname{Re} \lambda<\eta$ with values in $\mathrm{L}^{2}(G)$ and satisfies the boundedness condition

$$
\mathcal{N}_{G}^{0}\left(V,\left[\eta^{\prime}, \eta\right]\right)<\infty
$$

b) Conversely, let $U$ be a meromorphic function with values in $\mathrm{L}^{2}(G)$, such that $V$ defined by (3.10) is holomorphic in the strip $\eta^{\prime}<\operatorname{Re} \lambda<\eta$ and satisfies the boundedness condition (3.11). Then, like in the holomorphic case, the mapping (3.7) has limits at $\eta$ and $\eta^{\prime}$, and the inverse Mellin formulas (3.8) define $u \in \mathrm{K}_{\beta}^{0}(K)$ and $u^{\prime} \in \mathrm{K}_{\beta^{\prime}}^{0}(K)$. They satisfy the relation (3.9), which can be also written in the form of a residue formula:

$$
u(\mathbf{x})-u^{\prime}(\mathbf{x})=\frac{1}{2 i \pi} \int_{\mathfrak{C}^{c}} r^{\lambda} U(\lambda) \mathrm{d} \lambda,
$$

for a contour $\mathfrak{C}$ surrounding $\lambda_{0}$ and contained in the strip $\eta^{\prime}<\operatorname{Re} \lambda<\eta$. 
MELLIN ANALYSIS OF WEIGHTED SOBOLEV SPACES WITH NONHOMOGENEOUS NORMS ON CONES9

3.2. Mellin characterization of semi-norms. The principle of our Mellin analysis is to apply to a function $u$ and some of its derivatives $\partial_{\mathbf{x}}^{\alpha} u$ the Mellin characterization of Kweighted spaces from Theorems 3.2, 3.3, and 3.4.

\section{Definition 3.5.}

$\star$ For any $\alpha \in \mathbb{N}^{n}$, we denote by $\mathscr{D}^{\alpha}$ the differential operator in polar coordinates satisfying

$$
r^{|\alpha|} \partial_{\mathbf{x}}^{\alpha}=\mathscr{D}^{\alpha}\left(\vartheta ; r \partial_{r}, \partial_{\vartheta}\right)
$$

$\star$ For any $m \in \mathbb{N}$ and $\lambda \in \mathbb{C}$, let the parameter dependent semi-norm $|\cdot|_{m ; G ; \mathscr{D}(\lambda)}$ be defined on $\mathrm{H}^{m}(G)$ by

$$
|V|_{m ; G ; \mathscr{D}(\lambda)}^{2}=\sum_{|\alpha|=m}\left\|\mathscr{D}^{\alpha}\left(\vartheta ; \lambda, \partial_{\vartheta}\right) V\right\|_{0 ; G}^{2} .
$$

Lemma 3.6. Let $\beta<\beta_{0}$ be two real numbers. We set $\eta=-\beta-\frac{n}{2}$ and $\eta_{0}=-\beta_{0}-\frac{n}{2}$. Let $m \in \mathbb{N}$. Let $u \in \mathrm{K}_{\beta_{0}}^{0}(K)$ with support in $B(\mathbf{0}, 1)$ such that its $\mathrm{K}_{\beta}^{m}(K)$ semi-norm is finite.

Then the Mellin transform of $u$ is holomorphic for $\operatorname{Re} \lambda<\eta_{0}$ and has a meromorphic extension $U$ to the half-plane $\operatorname{Re} \lambda<\eta$. Its poles are contained in the set of integers

$$
\{0, \ldots, m-1\} \cap\left(\eta_{0}, \eta\right)
$$

and $U$ satisfies the estimates, with two constants $c, C>0$ independent of $u$

$$
c|u|_{\mathrm{K}_{\beta}^{m}(K)} \leqslant \sup _{b \in\left(\eta_{0}, \eta\right)}\left(\int_{\operatorname{Re} \lambda=b}|U(\lambda)|_{m ; G ; \mathscr{D}(\lambda)}^{2} \mathrm{~d} \operatorname{Im} \lambda\right)^{\frac{1}{2}} \leqslant C|u|_{\mathrm{K}_{\beta}^{m}(K)} .
$$

Proof. As $u \in \mathrm{K}_{\beta_{0}}^{0}(K)$, by Theorem 3.2 its Mellin transform $\lambda \mapsto \mathscr{M}[u](\lambda)$ is defined for all $\lambda$ on the line $\operatorname{Re} \lambda=\eta_{0}$. We set

$$
v_{m}:=r^{m} \partial_{r}^{m} u \quad \text { and } \quad w_{\alpha}:=r^{m} \partial_{\mathbf{x}}^{\alpha} u,|\alpha|=m .
$$

By assumption, the functions $w_{\alpha}$ for $|\alpha|=m$ all belong to $\mathrm{K}_{\beta}^{0}(K)$. Using the identity $r^{k} \partial_{r}^{k}=\sum_{|\beta|=k} \frac{k !}{\beta !} \mathbf{x}^{\beta} \partial_{\mathbf{x}}^{\beta}$, we obtain

$$
v_{m}=\sum_{|\alpha|=m} \frac{m !}{\alpha !} \vartheta^{\alpha} w_{\alpha}, \quad \text { with } \quad \vartheta^{\alpha}=\frac{\mathbf{x}^{\alpha}}{r^{m}},
$$

hence $v_{m}$ belongs to $\mathrm{K}_{\beta}^{0}(K)$ too. Therefore the Mellin transforms $\lambda \mapsto \mathscr{M}\left[v_{m}\right](\lambda)$ and $\lambda \mapsto \mathscr{M}\left[w_{\alpha}\right](\lambda)$ are defined for all $\lambda$ on the line $\operatorname{Re} \lambda=\eta$, and we have the estimates

$$
c|u|_{\mathrm{K}_{\beta}^{m}(K)}^{2} \leqslant \int_{\operatorname{Re} \lambda=\eta}\left(\left\|\mathscr{M}\left[v_{m}\right](\lambda)\right\|_{0 ; G}^{2}+\sum_{|\alpha|=m}\left\|\mathscr{M}\left[w_{\alpha}\right](\lambda)\right\|_{0 ; G}^{2}\right) \mathrm{d} \operatorname{Im} \lambda
$$

and

$$
\int_{\operatorname{Re} \lambda=\eta}\left(\left\|\mathscr{M}\left[v_{m}\right](\lambda)\right\|_{0 ; G}^{2}+\sum_{|\alpha|=m}\left\|\mathscr{M}\left[w_{\alpha}\right](\lambda)\right\|_{0 ; G}^{2}\right) \mathrm{d} \operatorname{Im} \lambda \leqslant C|u|_{\mathrm{K}_{\beta}^{m}(K)}^{2} .
$$

Since $u$, and thus $v_{m}$ and $w_{\alpha}$, have compact support, their Mellin transforms extend holomorphically to the half-planes $\operatorname{Re} \lambda<\eta_{0}$ for $u$, and $\operatorname{Re} \lambda<\eta$ for $v_{m}$ and $w_{\alpha}$. Moreover, 
due to the condition of support, estimate (3.16b) holds with the same constant $C$ if we replace the integral over the line $\operatorname{Re} \lambda=\eta$ with the integral over any line $\operatorname{Re} \lambda=b$, $\eta_{0}<b<\eta$ :

$$
\sup _{b \in\left(\eta_{0}, \eta\right)} \int_{\operatorname{Re} \lambda=b}\left(\left\|\mathscr{M}\left[v_{m}\right](\lambda)\right\|_{0 ; G}^{2}+\sum_{|\alpha|=m}\left\|\mathscr{M}\left[w_{\alpha}\right](\lambda)\right\|_{0 ; G}^{2}\right) \mathrm{d} \operatorname{Im} \lambda \leqslant C|u|_{\mathrm{K}_{\beta}^{m}(K)}^{2} .
$$

Using the identity

$$
r^{m} \partial_{r}^{m}=r \partial_{r}\left(r \partial_{r}-1\right) \cdots\left(r \partial_{r}-m+1\right),
$$

we find for all $\lambda, \operatorname{Re} \lambda \leqslant \eta_{0}$, the following relation between Mellin transforms:

$$
\mathscr{M}\left[v_{m}\right](\lambda)=\lambda(\lambda-1) \cdots(\lambda-m+1) \mathscr{M}[u](\lambda) .
$$

Hence we define a meromorphic extension $U$ of $\mathscr{M}[u]$ by setting

$$
U(\lambda)=\frac{\mathscr{M}\left[v_{m}\right](\lambda)}{\lambda(\lambda-1) \cdots(\lambda-m+1)} \quad \text { for } \quad \operatorname{Re} \lambda \leqslant \eta .
$$

Since $\mathscr{M}\left[w_{\alpha}\right](\lambda)=\mathscr{D}^{\alpha}\left(\vartheta ; \lambda, \partial_{\vartheta}\right) \mathscr{M}[u](\lambda)$ for Re $\lambda \leqslant \eta_{0}$, by meromorphic extension we find that

$$
\mathscr{M}\left[w_{\alpha}\right](\lambda)=\mathscr{D}^{\alpha}\left(\vartheta ; \lambda, \partial_{\vartheta}\right) U(\lambda), \text { for } \quad \operatorname{Re} \lambda \leqslant \eta .
$$

Putting (3.16a), (3.17), (3.20) together and using the semi-norm $|\cdot|_{m ; G ; \mathscr{D}(\lambda)}$ we have proved the equivalence (3.15).

In Theorem 3.12 below we will see that under the conditions of the Lemma, the poles of the Mellin transform of $u$ are associated with polynomials, corresponding to the Taylor expansion of $u$ at the origin.

If $\lambda$ is not an integer in the interval $[0, m-1]$, the semi-norm $|V|_{m ; G ; \mathscr{D}(\lambda)}$ defines a norm on $\mathrm{H}^{m}(G)$ equivalent to the parameter dependent norm $\|V\|_{m ; G ; \lambda}$ introduced in Definition 3.1. In order to describe this equivalence in a neighborhood of integers, we need to introduce a projection operator on polynomial traces on $G$ :

Definition 3.7. Let $k \in \mathbb{N}$.

* By $\mathrm{P}^{k}(G)$ we denote the space of restrictions to $G$ of homogeneous polynomial functions of degree $k$ on $K$.

* Let $\left(\varphi_{\gamma}^{k}\right)_{|\gamma|=k}$ be the basis in $\mathrm{P}^{k}(G)$ dual in $\mathrm{L}^{2}(G)$ of the homogeneous monomials $\left(\vartheta^{\alpha} / \alpha !\right)_{|\alpha|=k}\left(\vartheta=\frac{\mathbf{x}}{|\mathbf{x}|}\right)$, i.e.:

$$
\int_{G} \frac{\vartheta^{\alpha}}{\alpha !} \varphi_{\gamma}^{k}(\vartheta) \mathrm{d} \vartheta=\delta_{\alpha \gamma}, \quad|\alpha|=|\gamma|=k .
$$

By $\mathfrak{P}^{k}$ we denote the projection operator $\mathrm{L}^{2}(G) \rightarrow \mathrm{P}^{k}(G)$ defined as

$$
\mathfrak{P}^{k} U=\sum_{|\alpha|=k}\left\langle U, \varphi_{\alpha}^{k}\right\rangle_{G} \frac{\vartheta^{\alpha}}{\alpha !}
$$

Lemma 3.8. Let $m \in \mathbb{N}$ and $\eta_{0}$, $\eta$ real numbers such that $\eta_{0}<0 \leqslant m<\eta$. Let $\delta \in\left(0, \frac{1}{2}\right)$. Then there exist two constants $C, c>0$ such that for all $V \in \mathrm{H}^{m}(G)$ the following estimates hold: 
MELLIN ANALYSIS OF WEIGHTED SOBOLEV SPACES WITH NONHOMOGENEOUS NORMS ON CONES1

a) For $\lambda$ satisfying $\operatorname{Re} \lambda \in\left[\eta_{0}, \eta\right]$ and $|\lambda-k| \geqslant \delta$ for all $k \in\{0, \ldots, m-1\}$ :

$$
c|V|_{m ; G ; \mathscr{D}(\lambda)} \leqslant\|V\|_{m ; G ; \lambda} \leqslant C|V|_{m ; G ; \mathscr{D}(\lambda)} .
$$

b) For $\lambda$ satisfying $|\lambda-k| \leqslant \delta$ for a $k \in\{0, \ldots, m-1\}$ :

$$
c|V|_{m ; G ; \mathscr{D}(\lambda)} \leqslant\left\|V-\mathfrak{P}^{k} V\right\|_{m ; G}+|\lambda-k|\left\|\mathfrak{P}^{k} V\right\|_{m ; G} \leqslant C|V|_{m ; G ; \mathscr{D}(\lambda)} .
$$

Proof. Let $A$ be an annulus of the form $\left\{\mathbf{x} \in K, \frac{1}{R}<|\mathbf{x}|<R\right\}$ for a $R>1$. It is not hard to see that one has the following equivalence of the norm $\|\cdot\|_{m ; G ; \lambda}$ and seminorm $|\cdot|_{m ; G ; \mathscr{D}(\lambda)}$ with the norm and semi-norm of $\mathrm{H}^{m}(A)$ on its closed subspace $\overline{\mathrm{S}}_{m}^{\lambda}(A)$ of homogeneous functions of the form $r^{\lambda} V(\vartheta)$ :

$$
\begin{aligned}
& c\left\|r^{\lambda} V\right\|_{m ; A} \leqslant\|V\|_{m ; G ; \lambda} \leqslant C\left\|r^{\lambda} V\right\|_{m ; A} \\
& c\left|r^{\lambda} V\right|_{m ; A} \leqslant|V|_{m ; G ; \mathscr{D}(\lambda)} \leqslant C\left|r^{\lambda} V\right|_{m ; A} .
\end{aligned}
$$

Here the equivalence constants can be chosen uniformly for $\lambda$ in the whole strip $\eta_{0} \leqslant$ $\operatorname{Re} \lambda \leqslant \eta$.

- The well-known Bramble-Hilbert lemma implies that the semi-norm $|\cdot|_{m ; A}$ is equivalent to the norm $\|\cdot\|_{m ; A}$ on $\overline{\mathrm{S}}_{m}^{\lambda}(A)$ if and only if $\overline{\mathrm{S}}_{m}^{\lambda}(A)$ does not contain any non-zero polynomial of degree $\leqslant m-1$ : Thus for all $\lambda \notin\{0, \ldots, m-1\}$ there exists $C_{\lambda}$ such that

$$
\left\|r^{\lambda} V\right\|_{m ; A} \leqslant C_{\lambda}\left|r^{\lambda} V\right|_{m ; A},
$$

and $C_{\lambda}$ can be chosen uniformly on the set $\operatorname{Re} \lambda \in\left[\eta_{0}, \eta\right]$ with $|\lambda-k| \geqslant \delta$ for all $k \in$ $\{0, \ldots, m-1\}$; whence estimates (3.23) in case a) of the lemma.

- Let $\lambda$ be such that $|\lambda-k| \leqslant \delta$ for a $k \in\{0, \ldots, m-1\}$. The left inequality in (3.24) is easy to prove with the help of the estimate

$$
\left|\mathfrak{P}^{k} V\right|_{m ; G ; \mathscr{D}(\lambda)} \leqslant C|\lambda-k|\left\|\mathfrak{P}^{k} V\right\|_{m ; G},
$$

which is follows from $\left|\mathfrak{P}^{k} V\right|_{m ; G ; \mathscr{D}(k)}=0$.

Concerning the right-hand-side estimate of (3.24), the Bramble-Hilbert lemma argument implies the equivalence of the semi-norm with the norm for functions $V$ such that $\mathfrak{P}^{k} V=$ 0 : For all $V \in \mathrm{H}^{m}(G)$ there holds

$$
\left\|V-\mathfrak{P}^{k} V\right\|_{m ; G} \leqslant C\left|V-\mathfrak{P}^{k} V\right|_{m ; G ; \mathscr{D}(\lambda)} .
$$

On the other hand, the operator $r^{m} \partial_{r}^{m}$ is a linear combination of the operators $r^{m} \partial_{\mathbf{x}}^{\alpha},|\alpha|=$ $m$, with coefficients bounded on $G$. Therefore

$$
\left\|r^{m} \partial_{r}^{m}\left(r^{\lambda} V\right)\right\|_{0 ; A} \leqslant C\left|r^{\lambda} V\right|_{m ; A} .
$$

From (3.18) we get $|\lambda(\lambda-1) \cdots(\lambda-m+1)|\|V\|_{0 ; G} \leqslant C\left\|r^{m} \partial_{r}^{m}\left(r^{\lambda} V\right)\right\|_{0 ; A}$, hence

$$
|\lambda-k|\|V\|_{0 ; G} \leqslant C\left\|r^{m} \partial_{r}^{m}\left(r^{\lambda} V\right)\right\|_{0 ; A} \leqslant C^{\prime}|V|_{m ; G ; \mathscr{D}(\lambda)} .
$$

From the continuity of $\mathfrak{P}^{k}$ in $\mathrm{L}^{2}(G)$, we deduce

$$
|\lambda-k|\left\|\mathfrak{P}^{k} V\right\|_{0 ; G} \leqslant C|V|_{m ; G ; \mathscr{D}(\lambda)} .
$$


The equivalence of norms in $L^{2}(G)$ and $\mathrm{H}^{m}(G)$ on the finite dimensional range of $\mathfrak{P}^{k}$ yields finally

$$
|\lambda-k|\left\|\mathfrak{P}^{k} V\right\|_{m ; G} \leqslant C|V|_{m ; G ; \mathscr{D}(\lambda)} .
$$

It remains to bound $\left\|V-\mathfrak{P}^{k} V\right\|_{m ; G}$. Using (1), (2) and (3) we find

$$
\begin{aligned}
\left\|V-\mathfrak{P}^{k} V\right\|_{m ; G} & \leqslant C\left|V-\mathfrak{P}^{k} V\right|_{m ; G ; \mathscr{D}(\lambda)} \\
& \leqslant C\left(|V|_{m ; G ; \mathscr{D}(\lambda)}+\left|\mathfrak{P}^{k} V\right|_{m ; G ; \mathscr{D}(\lambda)}\right) \\
& \leqslant C\left(|V|_{m ; G ; \mathscr{D}(\lambda)}+|\lambda-k|\left\|\mathfrak{P}^{k} V\right\|_{m ; G}\right) \\
& \leqslant C|V|_{m ; G ; \mathscr{D}(\lambda)},
\end{aligned}
$$

which completes the proof of the lemma.

Putting the norm equivalences (3.23) and (3.24) together, one is led to the following definition of norms of meromorphic $\mathrm{H}^{m}(G)$-valued functions.

Definition 3.9. Let $\lambda \mapsto U(\lambda)$ be a meromorphic function with values in $\mathrm{H}^{m}(G)$ for $\lambda$ in a strip $b_{0}<\operatorname{Re} \lambda<b_{1}$.

$\star$ For $b \in\left(b_{0}, b_{1}\right)$ and $k \in \mathbb{N}$, and with $\mathfrak{P}_{G}^{k}$ the projection operator (3.22) we set

$$
\begin{aligned}
\mathcal{N}_{G}^{m}(U, b, k)= & \left\{\int_{\substack{|\operatorname{Im} \lambda| \leqslant 1 \\
\operatorname{Re} \lambda=b}}\left\|\left(\mathbb{I}-\mathfrak{P}_{G}^{k}\right) U(\lambda)\right\|_{m ; G}^{2} \mathrm{~d} \operatorname{Im} \lambda\right. \\
& +\int_{\substack{|\operatorname{Im} \lambda| \leqslant 1 \\
\operatorname{Re} \lambda=b}}|\lambda-k|^{2}\left\|\mathfrak{P}_{G}^{k} U(\lambda)\right\|_{m ; G}^{2} \mathrm{~d} \operatorname{Im} \lambda \\
& \left.+\int_{\substack{|\operatorname{Im} \lambda| \geqslant 1 \\
\operatorname{Re} \lambda=b}}\|U(\lambda)\|_{m ; G ; \lambda}^{2} \mathrm{~d} \operatorname{Im} \lambda\right\}^{\frac{1}{2}}
\end{aligned}
$$

$\star$ For $\mathfrak{N}=\left\{k_{1}, \ldots, k_{j}\right\} \subset \mathbb{N} \cap\left[b_{0}, b_{1}\right]$, and using the norm (3.4), we introduce

$$
\begin{aligned}
\mathcal{N}_{G}^{m}\left(U,\left[b_{0}, b_{1}\right], \mathfrak{N}\right) & = \\
\max & \left\{\sup _{b \in B_{0}} \mathcal{N}_{G}^{m}(U, b), \sup _{b \in B_{1}} \mathcal{N}_{G}^{m}\left(U, b, k_{1}\right), \ldots, \sup _{b \in B_{j}} \mathcal{N}_{G}^{m}\left(U, b, k_{j}\right)\right\},
\end{aligned}
$$

with the sets $B_{\ell}=\left(k_{\ell}-\frac{1}{2}, k_{\ell}+\frac{1}{2}\right) \cap\left(b_{0}, b_{1}\right)$ for $\ell=1, \ldots, j$ and

$$
B_{0}=\left(b_{0}, b_{1}\right) \backslash \cup_{\ell=1}^{j} B_{\ell} .
$$

* If $\mathfrak{N}=\emptyset$, the definition (3.25b) becomes, compare Definition 3.1,

$$
\mathcal{N}_{G}^{m}\left(U,\left[b_{0}, b_{1}\right], \emptyset\right)=\sup _{b \in\left(b_{0}, b_{1}\right)} \mathcal{N}_{G}^{m}(U, b)=\mathcal{N}_{G}^{m}\left(U,\left[b_{0}, b_{1}\right]\right)
$$

Using the continuity of $\mathfrak{P}_{G}^{k}$ on $\mathrm{H}^{m}(G)$, we obtain the estimate

$$
\mathcal{N}_{G}^{m}(U, b, k) \leqslant C_{b, m, k} \mathcal{N}_{G}^{m}(U, b)
$$


where the constant $C_{b, m, k}$ does not depend on $U$. On the other hand, the definition immediately implies the estimate

$$
\mathcal{N}_{G}^{m}(U, b) \leqslant(b-k)^{-1} \mathcal{N}_{G}^{m}(U, b, k), \quad \text { if } b \neq k .
$$

A consequence of the latter two inequalities is that for any fixed real number $\rho \in\left(0, \frac{1}{2}\right]$, we would obtain an equivalent norm to (3.25b) by defining $B_{\ell}$ as $\left(k_{\ell}-\rho, k_{\ell}+\rho\right) \cap\left(b_{0}, b_{1}\right)$ instead of $\left(k_{\ell}-\frac{1}{2}, k_{\ell}+\frac{1}{2}\right) \cap\left(b_{0}, b_{1}\right)$.

3.3. Spaces defined by Mellin norms. The norms defined in (3.25b) suggest the introduction of a class of Sobolev spaces $\mathrm{N}_{\beta, \beta_{0} ; \mathfrak{N}}^{m}$ with Mellin transforms meromorphic in a strip $\eta_{0}<\operatorname{Re} \lambda<\eta$ and a fixed set of poles $\mathfrak{N}$.

Definition 3.10. Let $m \in \mathbb{N}$ and $\beta, \beta_{0} \in \mathbb{R}$ such that $\beta \leqslant \beta_{0}$ and as usual, $\eta=-\beta-\frac{n}{2}$, $\eta_{0}=-\beta_{0}-\frac{n}{2}$. Let $\mathfrak{N}$ be a subset of $\mathbb{N} \cap\left[\eta_{0}, \eta\right]$.

$\star$ The functions $u \in \mathrm{N}_{\beta, \beta_{0} ; \mathfrak{N}}^{m}(K)$ with support in $B(\mathbf{0}, 1)$ are the functions whose Mellin transform $\mathscr{M}[u]$ is holomorphic in the half-plane $\operatorname{Re} \lambda<\eta_{0}$ and has a meromorphic extension $U$ to the half-plane $\operatorname{Re} \lambda<\eta$ satisfying the estimate

$$
\mathcal{N}_{G}^{m}\left(U,\left[\eta_{0}, \eta\right], \mathfrak{N}\right)<\infty .
$$

Let $\chi \in \mathscr{C}^{\infty}\left(\mathbb{R}^{\nu}\right)$ be a cut-off function with support in $B(\mathbf{0}, 1)$, equal to 1 in a neighborhood of the origin. The elements $u$ of $\mathrm{N}_{\beta, \beta_{0} ; \mathfrak{N}}^{m}(S)$ are defined by the two conditions that $\chi u \in \mathrm{N}_{\beta, \beta_{0} ; \mathfrak{N}}^{m}(K)$ and $(1-\chi) u \in \mathrm{H}^{m}(S)$.

* In the case when $\eta_{0}=\min \{0, \eta\}$ and $\mathfrak{N}=\mathbb{N} \cap\left[\eta_{0}, \eta\right]$, the space $\mathrm{N}_{\beta, \beta_{0} ; \mathfrak{N}}^{m}(K)$ will alternatively be denoted by $\mathrm{J}_{\max , \beta}^{m}(K)$.

Note that in this definition, the set of poles $\mathfrak{N}$ is contained in the interval $\left[\eta_{0}, \eta\right]$ determined by the weight exponents, but $\mathfrak{N}$ has no relation with the regularity order $m$. Thus the residues at the poles which, according to Theorem 3.4, give an asymptotic expansion at the origin, can only be identified with the terms of a Taylor expansion in a generalized sense, in general, because the corresponding derivatives need not exist outside of the origin. With $m=0$, for example, one gets weighted $\mathrm{L}^{2}$ spaces with detached asymptotics.

For the maximal J-weighted Sobolev spaces $J_{\max , \beta}^{m}$, the definition immediately yields the following properties.

Proposition 3.11. a) For all $m \geqslant 0, \beta<\beta^{\prime}$ implies $\mathrm{J}_{\max , \beta}^{m}(S) \subset \mathrm{J}_{\max , \beta^{\prime}}^{m}(S)$.

b) For all $\beta \in \mathbb{R}, 0 \leqslant m^{\prime}<m$ implies $\mathrm{J}_{\max , \beta}^{m}(S) \subset \mathrm{J}_{\max , \beta}^{m^{\prime},}(S)$.

c) $\partial_{\mathbf{x}}^{\alpha}$ is continuous from $\mathrm{J}_{\max , \beta}^{m}(S)$ into $\mathrm{J}_{\max , \beta+|\alpha|}^{m-|\alpha|}(S)$ for any $\alpha \in \mathbb{N}^{n}$, any $m \geqslant|\alpha|$, and any $\beta$.

d) The multiplication by $\mathbf{x}^{\alpha}$ is continuous from $\mathrm{J}_{\max , \beta}^{m}(S)$ into $\mathrm{J}_{\max , \beta-|\alpha|}^{m}(S)$ for any $\alpha \in \mathbb{N}^{n}$.

From Definition 3.10 follows that the poles of the Mellin transform of elements of $\mathrm{N}_{\beta, \beta_{0} ; \mathfrak{N}}^{m}$ are associated with polynomials and that $\mathrm{N}_{\beta, \beta_{0} ; \mathfrak{N}}^{m}$ can be split into a sum of a space with homogeneous norm and a space of polynomials. 
Theorem 3.12. Let $m \in \mathbb{N}$ and $\beta, \beta_{0} \in \mathbb{R}$ such that $\beta \leqslant \beta_{0}$ and as usual, $\eta=-\beta-\frac{n}{2}$, $\eta_{0}=-\beta_{0}-\frac{n}{2}$. Let $\mathfrak{N}$ be a subset of $\mathbb{N} \cap\left[\eta_{0}, \eta\right]$. Let $u \in \mathbb{N}_{\beta, \beta_{0} ; \mathfrak{N}}^{m}(K)$ with support in $B(0,1)$ and let $U$ be its Mellin transform. Then for $b \in\left(\eta_{0}, \eta\right] \backslash \mathfrak{N}$, the inverse Mellin transform $u^{\prime}$ of $U$ on the line $\operatorname{Re} \lambda=b$ belongs to $\mathrm{K}_{-b-\frac{n}{2}}^{m}(K)$ and there holds

$$
u^{\prime}-u=\sum_{k \in \mathfrak{N} \cap\left(\eta_{0}, b\right)} \operatorname{Res}_{\lambda=k}\left\{r^{\lambda} U(\lambda)\right\} \quad \text { is a polynomial. }
$$

The coefficients of the polynomial in (3.28) depend continuously on $u$ in the norm of $\mathrm{N}_{\beta, \beta_{0} ; \mathfrak{N}}^{m}(K)$.

Proof. Let $b \in\left(\eta_{0}, \eta\right] \backslash \mathfrak{N}$. By definition of $\mathrm{N}_{\beta, \beta_{0} ; \mathfrak{N}}^{m}(K)$, we have in particular

$$
\mathcal{N}_{G}^{m}(U, b)=\left(\int_{\operatorname{Re} \lambda=b}\|U(\lambda)\|_{m ; G ; \lambda}^{2} \mathrm{~d} \operatorname{Im} \lambda\right)^{\frac{1}{2}}<\infty .
$$

Theorem 3.2 then provides the existence of a function $u^{\prime} \in \mathrm{K}_{-b-\frac{n}{2}}^{m}(K)$ such that

$$
\mathscr{M}\left[u^{\prime}\right](\lambda)=U(\lambda), \quad \forall \lambda, \operatorname{Re} \lambda=b,
$$

and according to Theorem 3.4, there holds

$$
u^{\prime}-u=\frac{1}{2 i \pi} \int_{\mathfrak{C}} r^{\lambda} U(\lambda) \mathrm{d} \lambda=\sum_{k \in \mathfrak{N} \cap\left(\eta_{0}, b\right)} \operatorname{Res}_{\lambda=k}\left\{r^{\lambda} U(\lambda)\right\} .
$$

Here $\mathfrak{C}$ is a contour surrounding the poles of $U$ in $\mathfrak{N} \cap\left[\eta_{0}, b\right]$.

It remains to show that the residual at $k \in \mathfrak{N} \cap\left(\eta_{0}, b\right)$ is a polynomial. From the finiteness of $\mathcal{N}_{G}^{m}\left(U,\left[\eta_{0}, \eta\right], \mathfrak{N}\right)$ follows in particular that

$$
\sup _{|b-k|<1 / 2} \mathcal{N}_{G}^{m}(U, b, k)<\infty
$$

and therefore that both $\left(\mathbb{I}-\mathfrak{P}_{G}^{k}\right) U(\lambda)$ and $(\lambda-k) \mathfrak{P}_{G}^{k} U(\lambda)$ are holomorphic at $k$. Hence

$$
\underset{\lambda=k}{\operatorname{Res}}\left\{r^{\lambda} U(\lambda)\right\}=\operatorname{Res}_{\lambda=k}\left\{r^{\lambda} \mathfrak{P}_{G}^{k} U(\lambda)\right\}=r^{k} \mathfrak{P}_{G}^{k} \operatorname{Res}_{\lambda=k} U(\lambda),
$$

which is a polynomial in $\mathbf{x}$ of degree $k$.

We can now complete the characterization of the $\mathrm{K}_{\beta}^{m}$ semi-norm that was begun in Lemma 3.6.

Theorem 3.13. Let $\beta<\beta_{0}$ be two real numbers. We set $\eta=-\beta-\frac{n}{2}, \eta_{0}=-\beta_{0}-\frac{n}{2}$ and $\mathfrak{N}_{m}=\{0, \ldots, m-1\} \cap\left(\eta_{0}, \eta\right]$. Let $u \in \mathrm{K}_{\beta_{0}}^{0}(K)$ with support in $B(\mathbf{0}, 1)$. Let $U$ be its Mellin transform.

(1) Then the following two conditions are equivalent

a. The semi-norm $|u|_{\mathrm{K}_{\beta}^{m}(K)}$ is finite.

b. $u \in \mathrm{N}_{\beta, \beta_{0} ; \mathfrak{N}_{m}}^{m}(K)$.

(2) Moreover we have the equivalence of norms

$$
\begin{aligned}
c\left(\|u\|_{\mathrm{K}_{\beta_{0}}^{0}(K)}+|u|_{\mathrm{K}_{\beta}^{m}(K)}\right) \leqslant \mathcal{N}_{G}^{m}\left(U,\left[\eta_{0}, \eta\right], \mathfrak{N}_{m}\right) & \\
& \leqslant C\left(\|u\|_{\mathrm{K}_{\beta_{0}}^{0}(K)}+|u|_{\mathrm{K}_{\beta}^{m}(K)}\right) .
\end{aligned}
$$


MELLIN ANALYSIS OF WEIGHTED SOBOLEV SPACES WITH NONHOMOGENEOUS NORMS ON CONES5

Proof. a. $\Rightarrow$ b. and equivalence (3.29). Let $u \in \mathrm{K}_{\beta_{0}}^{0}(K)$ with finite $\mathrm{K}_{\beta}^{m}(K)$ semi-norm and support in $B(\mathbf{0}, 1)$. According to Lemma 3.6 its Mellin transform is defined for $\operatorname{Re} \lambda \leqslant$ $\eta_{0}$ and has a meromorphic extension $U$ to the half-plane $\operatorname{Re} \lambda<\eta$ satisfying estimates (3.15), i.e., the semi-norm $|u|_{\mathrm{K}_{\beta}^{m}(K)}$ is equivalent to the norm

$$
\sup _{b \in\left(\eta_{0}, \eta\right)}\left(\int_{\operatorname{Re} \lambda=b}|U(\lambda)|_{m ; G ; \mathscr{D}(\lambda)}^{2} \mathrm{~d} \operatorname{Im} \lambda\right)^{\frac{1}{2}} .
$$

But Lemma 3.8 reveals that the norm (1) is equivalent to

$$
\mathcal{N}_{G}^{m}\left(U,\left[\eta_{0}, \eta\right], \overline{\mathfrak{N}}_{m}\right) \quad \text { with } \quad \overline{\mathfrak{N}}_{m}=\{0, \ldots, m-1\} \cap\left[\eta_{0}, \eta\right] .
$$

Hence Lemma 3.6 yields the equivalence of the norm (2) with the semi-norm $|u|_{\mathrm{K}_{\beta}^{m}(K)}$.

On the other hand, the norm $\|u\|_{\mathrm{K}_{\beta_{0}}^{0}(K)}$ is equivalent to $\mathcal{N}_{G}^{0}\left(U, \eta_{0}\right)$. Therefore the norm

$$
\|u\|_{\mathrm{K}_{\beta_{0}}^{0}(K)}+|u|_{\mathrm{K}_{\beta}^{m}(K)}
$$

present in (3.29) is equivalent to

$$
\mathcal{N}_{G}^{0}\left(U, \eta_{0}\right)+\mathcal{N}_{G}^{m}\left(U,\left[\eta_{0}, \eta\right], \overline{\mathfrak{N}}_{m}\right) .
$$

It remains to prove that (4) is equivalent to $\mathcal{N}_{G}^{m}\left(U,\left[\eta_{0}, \eta\right], \mathfrak{N}_{m}\right)$.

- If $\mathfrak{N}_{m}=\overline{\mathfrak{N}}_{m}$ (this occurs if $\eta_{0} \notin\{0, \ldots, m-1\}$ ), we have the equality of norms $\mathcal{N}_{G}^{m}\left(U,\left[\eta_{0}, \eta\right], \mathfrak{N}_{m}\right)=\mathcal{N}_{G}^{m}\left(U,\left[\eta_{0}, \eta\right], \overline{\mathfrak{N}}_{m}\right)$. Moreover $\mathcal{N}_{G}^{0}\left(U, \eta_{0}\right)$ is bounded by the quantity $\mathcal{N}_{G}^{m}\left(U,\left[\eta_{0}, \eta\right], \mathfrak{N}_{m}\right)$. Hence the desired equivalence.

- If $\mathfrak{N}_{m} \neq \overline{\mathfrak{N}}_{m}$, then $\eta_{0} \in\{0, \ldots, m-1\}$ and

$$
\overline{\mathfrak{N}}_{m}=\mathfrak{N}_{m} \cup\left\{\eta_{0}\right\} .
$$

Since all norms are equivalent on the range of $\mathfrak{P}_{G}^{k}$, we find the estimate

$$
\mathcal{N}_{G}^{m}\left(U, \eta_{0}\right) \leqslant C\left(\mathcal{N}_{G}^{0}\left(U, \eta_{0}\right)+\mathcal{N}_{G}^{m}\left(U, \eta_{0}, \eta_{0}\right)\right) \leqslant C \cdot \operatorname{norm}(4) .
$$

Let us choose $b \in\left(\eta_{0}, \eta_{0}+\frac{1}{2}\right)$. We have

$$
\mathcal{N}_{G}^{m}(U, b) \leqslant C(b) \mathcal{N}_{G}^{m}\left(U,\left[\eta_{0}, \eta\right], \overline{\mathfrak{N}}_{m}\right),
$$

where $C(b)$ means that this constant depends on $b$ (and would blow up if $b$ approaches $\eta_{0}$, cf. (3.26b)). The finiteness of $\mathcal{N}_{G}^{m}\left(U, \eta_{0}\right)$ and $\mathcal{N}_{G}^{m}(U, b)$ implies that $u \in \mathrm{K}_{\beta_{0}}^{m}(K) \cap$ $\mathrm{K}_{-b-\frac{n}{2}}^{m}(K)$ and by Theorem 3.3:

$$
\mathcal{N}_{G}^{m}\left(U,\left[\eta_{0}, b\right], \emptyset\right) \leqslant C\left(\mathcal{N}_{G}^{m}\left(U, \eta_{0}\right)+\mathcal{N}_{G}^{m}(U, b)\right) .
$$

(6)-(8) gives that $\mathcal{N}_{G}^{m}\left(U,\left[\eta_{0}, b\right], \emptyset\right)$ is bounded by norm (4), which, in association with (5) implies that $\mathcal{N}_{G}^{m}\left(U,\left[\eta_{0}, \eta\right], \mathfrak{N}_{m}\right)$ is bounded by (4). The converse estimate is obvious.

b. $\Rightarrow$ a. Let $u \in \mathrm{K}_{\beta_{0}}^{0}(K) \cap \mathrm{N}_{\beta, \beta_{0} ; \mathfrak{N}_{m}}^{m}(K)$ with support in $B(\mathbf{0}, 1)$. Since $\eta_{0} \notin \mathfrak{N}_{m}$, we have in particular, cf. (3.26b)

$$
\mathcal{N}_{G}^{m}\left(U, \eta_{0}\right) \leqslant C \mathcal{N}_{G}^{m}\left(U,\left[\eta_{0}, \eta\right], \mathfrak{N}_{m}\right) .
$$


Hence we obtain that $u$ belongs to $\mathrm{K}_{\beta_{0}}^{m}(K)$. Therefore, for all $\alpha,|\alpha|=m$, the function $w_{\alpha}=r^{m} \partial_{\mathbf{x}}^{\alpha} u$ belongs to $\mathbf{K}_{\beta_{0}}^{0}(K)$. Let $W_{\alpha}$ be its Mellin transform. By construction, and thanks to Lemma 3.8, we find

$$
\mathcal{N}_{G}^{0}\left(W_{\alpha},\left[\eta_{0}, \eta\right], \emptyset\right) \leqslant C \mathcal{N}_{G}^{m}\left(U,\left[\eta_{0}, \eta\right], \mathfrak{N}_{m}\right)
$$

Hence $w_{\alpha} \in \mathrm{K}_{\beta}^{0}(K)$, and therefore the $\mathrm{K}_{\beta}^{m}(K)$ semi-norm of $u$ is finite.

3.4. Spaces defined by weighted semi-norms. We have seen in Theorem 3.13 how a space defined by two weighted seminorms $|\cdot|_{K_{\beta_{0}}^{0}}$ and $|\cdot|_{K_{\beta}^{m}}$ has a Mellin characterization described by the space $\mathrm{N}_{\beta, \beta_{0} ; \mathfrak{N}_{m}}^{m}$. We are now generalizing this to the case of spaces given by several weighted semi-norms, and this will eventually lead to the Mellin characterization of the space $\mathrm{J}_{\beta}^{m}$, which is defined by the $m+1$ semi-norms $|\cdot|_{\mathrm{K}_{\beta+m-\ell}^{\ell}}, 0 \leqslant \ell \leqslant m$, see Definition 2.2.

Definition 3.14. Let $\mathfrak{L}$ be a subset of $\mathbb{N}$ that includes 0 : For each $\ell \in \mathfrak{L}$ let $\beta_{\ell}$ be a weight exponent such that

$$
\beta_{\ell} \text { decreases as } \ell \text { increases, }
$$

and denote $\mathfrak{B}=\left\{\beta_{\ell}: \ell \in \mathfrak{L}\right\}$. We define the associated norm

$$
\|u\|_{J_{\mathfrak{B}}^{\mathfrak{L}}(S)}=\left(\sum_{\ell \in \mathfrak{L}} \sum_{|\alpha|=\ell}\left\|r^{\beta_{\ell}+\ell} \partial_{\mathbf{x}}^{\alpha} u\right\|_{0 ; S}^{2}\right)^{\frac{1}{2}} \equiv\left(\sum_{\ell \in \mathfrak{L}}|u|_{\mathrm{K}_{\beta_{\ell}}^{\ell}(S)}^{2}\right)^{\frac{1}{2}} .
$$

The Hilbert space defined by this norm is denoted by $\mathrm{J}_{\mathfrak{B}}^{\mathfrak{L}}(S)$.

This definition includes the weighted Sobolev spaces with homogeneous norms and those with non-homogeneous norms as obvious special cases:

- We obtain the norm in $\mathrm{K}_{\beta}^{m}$ by choosing $\beta_{\ell}=\beta$ for all $\ell$ and $\mathfrak{L}$ any arbitrary subset contained in $\{0, \ldots, m\}$ and containing 0 and $m$.

- According to Definition 2.2, we obtain the norm in $\mathrm{J}_{\beta}^{m}$ by choosing

$$
\mathfrak{L}=\{0, \ldots, m\}, \quad \beta_{\ell}=\beta+m-\ell .
$$

- Finally, the space defined by the norm $\mathrm{K}_{\beta_{0}}^{0}$ and the seminorm $\mathrm{K}_{\beta}^{m}$ simply corresponds to $\mathfrak{L}=\{0, m\}$ and $\mathfrak{B}=\left\{\beta_{0}, \beta\right\}$.

We can use Theorem 3.13 to obtain a first Mellin characterization of the space $\mathrm{J}_{\mathfrak{B}}^{\mathfrak{L}}(S)$. We set, as usual, $\eta_{\ell}=-\beta_{\ell}-\frac{n}{2}$. Then we have

$$
\mathrm{J}_{\mathfrak{B}}^{\mathfrak{L}}(S)=\bigcap_{\ell \in \mathfrak{L}} \mathbf{N}_{\beta_{\ell}, \beta_{0} ; \mathfrak{N}_{\ell}}^{\ell}(S) \quad \text { with } \mathfrak{N}_{\ell}=\{0, \ldots, \ell-1\} \cap\left(\eta_{0}, \eta_{\ell}\right]
$$

This can be simplified with the following result.

Lemma 3.15. Let $\mathfrak{L}, \mathfrak{B}$ and $\mathfrak{N}_{\ell}$ be as above. Let $m=\max \mathfrak{L}$. Then there exists a unique subset $\mathfrak{N} \subset \mathfrak{N}_{m}=\{0, \ldots, m-1\} \cap\left(\eta_{0}, \eta\right]$ such that there is a norm equivalence

$$
c \mathcal{N}_{G}^{m}\left(U,\left[\eta_{0}, \eta\right], \mathfrak{N}\right) \leqslant \max _{\ell \in \mathfrak{L}} \mathcal{N}_{G}^{\ell}\left(U,\left[\eta_{0}, \eta_{\ell}\right], \mathfrak{N}_{\ell}\right) \leqslant C \mathcal{N}_{G}^{m}\left(U,\left[\eta_{0}, \eta\right], \mathfrak{N}\right) .
$$


MELLIN ANALYSIS OF WEIGHTED SOBOLEV SPACES WITH NONHOMOGENEOUS NORMS ON CONE\$7

This set $\mathfrak{N}$ is given by

$$
\mathfrak{N}=\mathfrak{N}_{m} \backslash\left(\bigcup_{\ell \in \mathfrak{L}}\left[\ell, \eta_{\ell}\right]\right)
$$

Proof. Assume that $\mathfrak{N}$ is such that the norm equivalence (3.32) holds. Then any pole $k \in \mathfrak{N}$ that lies in an interval $\left(\eta_{0}, \eta_{\ell}\right]$ must appear as a pole in the corresponding set $\mathfrak{N}_{\ell}$ and vice versa. This means

$$
\mathfrak{N} \cap\left(\eta_{0}, \eta_{\ell}\right] \subset\{0, \ldots, \ell-1\} \quad \forall \ell \in \mathfrak{L} .
$$

In other words, for $k \in \mathfrak{N}_{m}$ there holds $k \notin \mathfrak{N}$ if and only if there exists $\ell \in \mathfrak{L}$ such that

$$
\ell \leqslant k \leqslant \eta_{\ell}
$$

This implies the formula (3.33) for $\mathfrak{N}$. Conversely, it is not hard to see that if we define the set $\mathfrak{N}$ by (3.33), then the norm equivalence (3.32) holds.

Combining (3.31) with Lemma 3.15, we obtain the Mellin characterization of the space $\mathrm{J}_{\mathfrak{B}}^{\mathfrak{L}}(S)$.

Proposition 3.16. Let $\mathfrak{L}$ and $\mathfrak{B}$ satisfy the conditions in Definition 3.14. Let $m=\max \mathfrak{L}$, $\beta=\beta_{m}$ and define $\mathfrak{N}$ by (3.33). Then

$$
\mathrm{J}_{\mathfrak{B}}^{\mathfrak{L}}(S)=\mathrm{N}_{\beta, \beta_{0} ; \mathfrak{N}}^{m}(S) .
$$

We have seen that with each set of semi-norms given by $\mathfrak{L}$ and $\mathfrak{B}$ there is a unique associated set of poles $\mathfrak{N}$ that characterizes the space $\mathrm{N}_{\beta, \beta_{0} ; \mathfrak{N}}^{m}(S)$ and therefore the space $\mathrm{J}_{\mathfrak{B}}^{\mathfrak{L}}(S)$. The converse is not always true, that is, the spaces $\mathrm{N}_{\beta, \beta_{0} ; \mathfrak{N}}^{m}$ cannot always be defined by a set of weighted Sobolev semi-norms. A necessary condition is that $\mathfrak{N} \subset \mathfrak{N}_{m}$. But this is also sufficient:

For fixed $m$ and $\beta$, let $\mathfrak{N}$ be a given subset of $\mathfrak{N}_{m}$. We can construct indices $\mathfrak{L}$ and weight exponents $\mathfrak{B}$ such that the formula (3.33), and therefore the equality of spaces (3.35) in Proposition 3.16 holds. This can be done by setting

$$
\mathfrak{L}=\{0\} \cup\left(\mathfrak{N}_{m} \backslash \mathfrak{N}\right) \cup\{m\},
$$

and for all $\ell \in \mathfrak{L}, \ell \neq 0, m$,

$$
\beta_{\ell}=-\eta_{\ell}-\frac{n}{2} \quad \text { with } \quad \eta_{\ell}=\ell,
$$

and $\eta_{0}=0$ if $0 \notin \mathfrak{N}, \eta_{0}<0$ arbitrary if $0 \in \mathfrak{N}$.

In this context, the counter-example (2.8), for instance, corresponds to the choice of $m \geqslant 2, \mathfrak{L}=\{0\} \cup\{2, \ldots, m\}$, and $\eta_{\ell}=\eta-m+\ell$ with $m<\eta<m+1$, so that $\eta_{\ell} \in(\ell, \ell+1)$. From these informations one obtains $\mathfrak{N}=\{1\}$.

From the equality (3.35), we conclude that the space $\mathrm{J}_{\mathfrak{B}}^{\mathfrak{L}}(S)$ depends only on $m, \beta=\beta_{m}$ and on the set of integers $\mathfrak{N}$. Several different choices of $\mathfrak{L}$ and $\mathfrak{B}$ can therefore lead to the same space. We have already seen this for the space $\mathrm{K}_{\beta}^{m}$, where the choice of $\mathfrak{L}$ is arbitrary, as soon as it includes 0 and $m$. This observation expresses the fact that for the spaces with homogeneous norms, the intermediate semi-norms are bounded by the two extreme semi-norms. The set of poles $\mathfrak{N}$ is empty in this case.

Also for the space with non-homogeneous norm $\mathrm{J}_{\beta}^{m}$, several different choices of sets of semi-norms are possible, as we will discuss now. 
3.5. Mellin characterization of spaces with non-homogeneous norms. For the weighted Sobolev space with non-homogeneous norm $\mathrm{J}_{\beta}^{m}$, several different choices of sets $\{\mathfrak{L}, \mathfrak{B}\}$ of semi-norms are possible that lead to the same set of poles $\mathfrak{N}$ and define therefore, according to Proposition 3.16, the same space. The original definition of $\mathrm{J}_{\beta}^{m}$ corresponds to the choice $\mathfrak{L}=\{0, \ldots, m\}$ and $\beta_{\ell}=\beta+m-\ell, \ell \in \mathfrak{L}$, which implies $\eta_{\ell+1}=\eta_{\ell}+1$. From this information and formula (3.33) one easily deduces that $\mathfrak{N}$ is either empty or a set of consecutive integers starting with 0 . It is non-empty if and only if $0<\eta<m$, and in this case

$$
\mathfrak{N}=\{0, \ldots, m-1\} \cap(\eta-m, \eta]=\{0, \ldots M\} \quad \text { with } M=[\eta] .
$$

Since $\mathfrak{N}=\emptyset$ corresponds to the space $\mathrm{K}_{\beta}^{m}$ and $\mathfrak{N}=\{0, \ldots,[\eta]\}$ to the space $\mathrm{J}_{\max , \beta}^{m}$, we have found the following classification of the space $\mathrm{J}_{\beta}^{m}$.

Proposition 3.17. Let $m \in \mathbb{N}, \beta \in \mathbb{R}$ and $\eta=-\beta-\frac{n}{2}$.

a) If $\eta<0$, then $\mathrm{J}_{\beta}^{m}(S)=\mathrm{J}_{\max , \beta}^{m}(S)=\mathrm{K}_{\beta}^{m}(S)$.

b) If $0 \leqslant \eta<m$, then $\mathrm{J}_{\beta}^{m}(S)=\mathrm{J}_{\max , \beta}^{m}(S)$.

c) If $\eta \geqslant m$, then $\mathrm{J}_{\beta}^{m}(S)=\mathrm{K}_{\beta}^{m}(S)$.

The set $\mathfrak{N}$ of integers (3.38) that characterizes $\mathrm{J}_{\beta}^{m}$ can also be obtained by other choices for the weight indices: We start again with $0<\eta<m$ and $\mathfrak{L}=\{0, \ldots, m\}$, but now we fix some integer $\ell_{0}$ in the interval $(\eta, m]$. Then we define the weight indices $\beta_{\ell}$ in such a way that

$$
\eta_{\ell}=\eta-\ell_{0}+\ell \quad \text { for } 0 \leqslant \ell \leqslant \ell_{0} \quad \text { and } \quad \eta_{\ell}=\eta \quad \text { for } \ell \geqslant \ell_{0}
$$

Since $\eta_{0}<0$ and $\ell_{0}-1 \geqslant M$, we easily see that this set of weight indices defines the same set of degrees $\mathfrak{N}=\{0, \ldots M\}$ as in (3.38). In this way, we have proved the "stepweighted" characterization of $\mathrm{J}_{\beta}^{m}$ :

Proposition 3.18. Let $\beta \in \mathbb{R}$ and $m \in \mathbb{N}$ such that $m>\eta=-\beta-\frac{n}{2}$. Let $\rho$ be any real number in the interval $\left(-\frac{n}{2}, \beta+m\right]$. Then the norm in the space $\mathrm{J}_{\beta}^{m}(S)$ is equivalent to

$$
\left(\sum_{|\alpha| \leqslant m}\left\|r^{\max \{\beta+|\alpha|, \rho\}} \partial_{\mathbf{x}}^{\alpha} u\right\|_{0 ; S}^{2}\right)^{\frac{1}{2}} .
$$

Corollary 3.19. Let $\beta \in \mathbb{R}$. Set $\eta=-\beta-\frac{n}{2}$. Let $m$ be a natural number, $m>\eta$. Then $\mathrm{J}_{\beta}^{m+1}(S) \subset \mathrm{J}_{\beta}^{m}(S)$.

Proof. Using Proposition 3.18, we note that we can choose the same $\rho$ for $\mathrm{J}_{\beta}^{m}(S)$ and $\mathrm{J}_{\beta}^{m+1}(S)$. The embedding $\mathrm{J}_{\beta}^{m+1}(S) \subset \mathrm{J}_{\beta}^{m}(S)$ follows.

Still another choice giving the same result is possible. When $0<\eta<m$, it suffices to take $\mathfrak{L}=\{0, m\}, \eta_{m}=\eta$, and any $\eta_{0}<0$. In this case,

$$
\mathfrak{N}=\mathfrak{N}_{m}=\{0, \ldots M\}
$$

which corresponds to the identity $\mathrm{J}_{\beta}^{m}(S)=\mathrm{J}_{\max , \beta}^{m}(S)$. We obtain the corollary that when $0<\eta<m$, the intermediate semi-norms in the definition of $\mathrm{J}_{\beta}^{m}(S)$ are indeed bounded by the sum of the two extreme semi-norms. This is not the case, as we have seen, if $\eta>m$. 
Let us mention another identity that can be obtained from these purely combinatorial arguments, namely

$$
\mathrm{K}_{\beta}^{m}(S)=\mathrm{J}_{\beta}^{m}(S) \cap \mathrm{K}_{\beta}^{0}(S) .
$$

This can be seen as follows: The intersection $\mathrm{J}_{\beta}^{m}(S) \cap \mathrm{K}_{\beta}^{0}(S)$ is included in the space $\mathrm{J}_{\mathfrak{B}}^{\mathfrak{L}}(S)$ with $\mathfrak{L}=\{0, m\}$ and $\beta_{0}=\beta_{m}=\beta$. Then $\mathfrak{N}=\emptyset$ and we find that this latter space coincides with $\mathrm{K}_{\beta}^{m}(S)$.

Remark 3.20. Corollary 3.19 gives a partial response to the question of how to define spaces $\mathrm{J}_{\beta}^{s}$ with non-integer Sobolev index $s$. If $[s]>\eta$, the natural idea is to define the space of index $s$ by Hilbert space interpolation between spaces with integer indices $[s]$ and $[s]+1$. The same possibility exists if $[s]+1 \leqslant \eta$, since for $m+1 \leqslant \eta$ the inclusion $\mathrm{J}_{\beta}^{m+1}(S) \subset \mathrm{J}_{\beta}^{m}(S)$ holds, too, because according to Proposition $3.17 \mathrm{c}$ ) the J-weighted spaces coincide with the $\mathrm{K}$-weighted spaces in this range.

For fixed weight $\beta$, both scales of spaces $\left(\mathrm{K}_{\beta}^{m}(S)\right)_{m \in \mathbb{N}}$ and $\left(\mathrm{J}_{\max , \beta}^{m}(S)\right)_{m \in \mathbb{N}}$ can be extended in a natural way by interpolation to scales with arbitrary real positive index. This definition, when extended by analogy to the $n$-1-dimensional conical manifold $\partial K$, is then also compatible with the trace operator, that is, the trace space of $\mathrm{K}_{\beta}^{m}(K)$ is $\mathrm{K}_{\beta+\frac{1}{2}}^{m-\frac{1}{2}}(\partial K)$ and similarly for the $J_{\max }$ scale.

There is, however, no natural definition of $\mathrm{J}_{\beta}^{s}$ for the remaining non-integer indices $s$ for which $[s] \leqslant \eta<[s]+1$. The problem is that if $m>\eta$, so that $\mathrm{J}_{\beta}^{m}(S)=\mathrm{J}_{\max , \beta}^{m}(S)$, then the trace space is also of the $J_{\max }$ class, because it contains non-zero constant functions. But if $m-\frac{1}{2}<\eta$, then the candidate for the trace space would be $\mathrm{J}_{\beta+\frac{1}{2}}^{m-\frac{1}{2}}$ and should be of the $\mathrm{K}$ class, which does not contain non-constant functions.

As a further corollary of the Mellin description of the space $\mathrm{J}_{\beta}^{m}$, we give an equivalent definition by derivatives in polar coordinates that is valid when $\eta<1$ and will be useful later on:

Lemma 3.21. Let $\beta \in \mathbb{R}, \eta=-\beta-\frac{n}{2}$ and $m \in \mathbb{N}, m \geqslant 1$. We assume that $\eta<1$. Then

$$
\left\{\sum_{1 \leqslant \ell+|\gamma| \leqslant m}\left\|r^{\beta}\left(r \partial_{r}\right)^{\ell} \partial_{\vartheta}^{\gamma} u\right\|_{0 ; S}^{2}+\left\|r^{\beta+1} u\right\|_{0, S}^{2}\right\}^{\frac{1}{2}}
$$

defines a norm on $\mathrm{J}_{\beta}^{m}(S)$, equivalent to its natural norm.

Proof. If $\eta<0$, the statement is clear because, in that case, $\mathrm{J}_{\beta}^{m}(S)$ coincides with $\mathrm{K}_{\beta}^{m}(S)$.

Let us suppose that $0 \leqslant \eta<1$ and let $u$ be such that its norm (3.40) is finite. Using a cut-off, we can assume that $u$ has the same regularity on $K$, with support in $\bar{S}$. Let $\mathscr{M}[u]=: U$ be the Mellin transform of $u$. By the Parseval identity we have the equivalence

$$
\sum_{1 \leqslant \ell+k \leqslant m} \int_{\operatorname{Re} \lambda=\eta}|\lambda|^{2 \ell}|U(\lambda)|_{k ; G}^{2} \mathrm{~d} \operatorname{Im} \lambda \simeq \sum_{1 \leqslant \ell+|\gamma| \leqslant m}\left\|r^{\beta}\left(r \partial_{r}\right)^{\ell} \partial_{\vartheta}^{\gamma} u\right\|_{0 ; S}^{2} .
$$

It is easy to see that we have the uniform estimate

$$
\sum_{k=1}^{m}|U(\lambda)|_{k ; G ; \mathscr{D}(\lambda)} \leqslant C \sum_{1 \leqslant \ell+k \leqslant m}|\lambda|^{\ell}|U(\lambda)|_{k ; G} .
$$


We deduce that $u \in \mathrm{J}_{\beta}^{m}(S)$.

Conversely, let $u \in \mathrm{J}_{\beta}^{m}(S)$. We apply Lemma 3.8. Outside a neighborhood of 0 , we have the uniform estimate:

$$
\|U(\lambda)\|_{m ; G ; \lambda} \leqslant C|U(\lambda)|_{m ; G ; \mathscr{D}(\lambda)},
$$

and in a bounded neighborhood of 0

$$
\left\|U(\lambda)-\mathfrak{P}^{0} U(\lambda)\right\|_{m ; G}+|\lambda|\|U(\lambda)\|_{m ; G} \leqslant C|U(\lambda)|_{m ; G ; \mathscr{D}(\lambda)} .
$$

Since $\mathfrak{P}^{0} U(\lambda)$ is a constant, there holds

$$
\sum_{k=1}^{m}|U(\lambda)|_{k ; G} \leqslant C\left\|U(\lambda)-\mathfrak{P}^{0} U(\lambda)\right\|_{m ; G} .
$$

We deduce from (2)-(4) that

$$
\sum_{1 \leqslant \ell+k \leqslant m}|\lambda|^{\ell}|U(\lambda)|_{k ; G} \leqslant C|U(\lambda)|_{m ; G ; \mathscr{D}(\lambda)} .
$$

The boundedness of norm (3.40) follows from (1) and Lemma 3.6.

We can now collect the informations about the Mellin description of the space $\mathrm{J}_{\beta}^{m}$. For this, we introduce some notation concerning the Taylor expansion at the origin:

For $u \in \mathscr{C}^{\infty}(\bar{S})$ and $M \in \mathbb{N}$, we write $\mathfrak{T}^{M} u \in \mathbb{P}^{M}(S)$ for the Taylor part of $u$ of degree $M$ at $\mathbf{0 :}$

$$
\mathfrak{T}^{M} u=\sum_{|\alpha| \leqslant M} \partial_{\mathbf{x}}^{\alpha} u(\mathbf{0}) \frac{\mathbf{x}^{\alpha}}{\alpha !} .
$$

By continuity, the coefficients of the Taylor expansion and therefore the corner Taylor operator $\mathfrak{T}^{M}$ can be defined on the space $\mathbb{N}_{\beta, \beta_{0} ; \mathfrak{N}}^{m}(S)$, as soon as $\{0, \ldots, M\} \subset \mathfrak{N} \subset$ $\left(\eta_{0}, \eta\right)$, see Theorem 3.12.

The proofs of the following two theorems are contained in the results of the preceding section.

Theorem 3.22. Let $K$ be a regular cone in $\mathbb{R}^{n}$. Let $\beta \in \mathbb{R}$. We set, as usual

$$
\eta=-\beta-\frac{n}{2} \quad \text { and } \quad M=[\eta] .
$$

Let $\mathfrak{N}=\{0, \ldots, M\}$ if $M \geqslant 0$ and $m>\eta$, and $\mathfrak{N}=\emptyset$ in the other cases (either $M<0$ or $m \leqslant \eta)$. Let $u \in \mathrm{K}_{\beta+m}^{0}(K)$ with support in $B(\mathbf{0}, 1)$. Let $U$ be its Mellin transform. Set $\eta_{0}=\eta-m$ and $\beta_{0}=\eta_{0}-\frac{n}{2}=\beta+m$.

a) Then $u \in J_{\beta}^{m}(K)$ if and only if $u \in \mathrm{N}_{\beta, \beta_{0} ; \mathfrak{N}}^{m}(K)$. Moreover we have the equivalence of norms

$$
c\|u\|_{J_{\beta}^{m}(K)} \leqslant \mathcal{N}_{G}^{m}(U,[\eta-m, \eta], \mathfrak{N}) \leqslant C\|u\|_{J_{\beta}^{m}(K)} .
$$

Furthermore $U$ is meromorphic in the half-plane $\operatorname{Re} \lambda<\eta$ with only possible poles on natural numbers and the residues of $r^{\lambda} U(\lambda)$ are polynomials. 
MELLIN ANALYSIS OF WEIGHTED SOBOLEV SPACES WITH NONHOMOGENEOUS NORMS ON CONES1

b) Let $M^{*}=M$ if $\eta \neq M$ and $M^{*}=M-1$ if $\eta=M$. Let $b \in\left(M^{*}, \eta\right]$ with $b \neq \eta$ if $\eta=M$. Then the inverse Mellin transform $u^{\prime}$ of $U$ on the line $\operatorname{Re} \lambda=b$ belongs to $\mathrm{K}_{-b-\frac{n}{2}}^{m}(K)$ and there holds, with notation (3.41)

$$
u^{\prime}-u=\sum_{k=0}^{M^{*}} \operatorname{Res}_{\lambda=k}\left\{r^{\lambda} U(\lambda)\right\}=-\mathfrak{T}^{M^{*}} u .
$$

When $M<0$ or $m \leqslant \eta$, the sum of residues collapses to 0 , and $u$ belongs to $\mathrm{K}_{\beta}^{m}(K)$.

We will call the case $\eta \in \mathbb{N}$ critical.

In the non-critical case, we can take $b=\eta$ in the previous result, and we obtain therefore the following relations between the space $\mathrm{J}_{\beta}^{m}(S)$ with non-homogeneous norm and the space $\mathrm{K}_{\beta}^{m}(S)$ with homogeneous norm:

Theorem 3.23. Let $K \subset \mathbb{R}^{n}$ be a cone and $S=K \cap B(\mathbf{0}, 1)$. Let $\beta \in \mathbb{R}$. We set

$$
\eta=-\beta-\frac{n}{2} \quad \text { and } \quad M=[\eta] .
$$

Let $m \in \mathbb{N}$. Then there holds:

a) If $\eta<0$, the spaces $\mathrm{J}_{\beta}^{m}(S)$ and $\mathrm{K}_{\beta}^{m}(S)$ coincide.

b) If $\eta \geqslant 0$ and $m \leqslant \eta$, the spaces $\mathrm{J}_{\beta}^{m}(S)$ and $\mathrm{K}_{\beta}^{m}(S)$ coincide.

c) If $\eta \geqslant 0$ and $m>\eta$, then $\mathrm{J}_{\beta}^{m}(S)$ and $\mathrm{J}_{\max , \beta}^{m}(S)$ coincide and there are two cases:

- The non-critical case $\eta \notin \mathbb{N}$ : The corner Taylor operator $\mathfrak{T}^{M}$ defined in (3.41) is continuous from $\mathrm{J}_{\beta}^{m}(S)$ to $\mathbb{P}^{M}(S)$ and $\mathbb{I}-\mathfrak{T}^{M}$ is continuous from $\mathrm{J}_{\beta}^{m}(S)$ to $\mathrm{K}_{\beta}^{m}(S)$.

The decomposition $u=\left(u-\mathfrak{T}^{M} u\right)+\mathfrak{T}^{M} u$ gives the direct sum

$$
\mathrm{J}_{\beta}^{m}(S)=\mathrm{K}_{\beta}^{m}(S) \oplus \mathbb{P}^{M}(S) .
$$

- The critical case $\eta \in \mathbb{N}$ : The operator $\mathfrak{T}^{M-1}$ is continuous on $\mathrm{J}_{\beta}^{m}(S)$, but $\mathfrak{T}^{M}$ is not. The space $\mathrm{J}_{\beta}^{m}(S)$ contains $\mathrm{K}_{\beta}^{m}(S) \oplus \mathbb{P}^{M}(S)$ as a strict subspace of infinite codimension.

The structure of $\mathrm{J}_{\beta}^{m}$ in the critical case, and the generalization of the Taylor expansion in that case, is the subject of the following section.

\section{STRUCTURE OF SPACES WITH NONHOMOGENEOUS NORMS IN THE CRITICAL CASE}

4.1. Weighted Sobolev spaces with analytic regularity. Using the monotonicity of embeddings $\mathrm{K}_{\beta}^{m+1}(S) \subset \mathrm{K}_{\beta}^{m}(S)$ for all $m$ and $\beta$ and $\mathrm{J}_{\beta}^{m+1}(S) \subset \mathrm{J}_{\beta}^{m}(S)$ if $M>\eta=-\beta-\frac{n}{2}$, we introduce corresponding weighted spaces with infinite and with analytic regularity:

Definition 4.1. Let $\beta \in \mathbb{R}$ and $\eta=-\beta-\frac{n}{2}$.

$$
\star \mathrm{K}_{\beta}^{\infty}(K)=\bigcap_{m \in \mathbb{N}} \mathrm{K}_{\beta}^{m}(K) .
$$


* We denote by $\mathrm{A}_{\beta}(K)$ the subspace of the functions $u \in \mathrm{K}_{\beta}^{\infty}(K)$ satisfying the following analytic estimates for some $C>0$

$$
\exists C>0, \quad \forall k \in \mathbb{N}, \quad|u|_{\mathrm{K}_{\beta}^{k}(K)} \leqslant C^{k+1} k ! .
$$

$\star \mathrm{J}_{\beta}^{\infty}(S)=\bigcap_{k \in \mathbb{N}, k>\eta} \mathrm{J}_{\beta}^{k}(S)$.

* The analytic weighted class $\mathrm{B}_{\beta}(S)$ with non-homogeneous norm is the space of functions $u \in \mathrm{J}_{\beta}^{\infty}(S)$ such that there exists a constant $C>0$ with

$$
\forall k \in \mathbb{N} \quad \text { with } \quad k>\eta, \quad|u|_{\mathrm{K}_{\beta}^{k}(K)} \leqslant C^{k+1} k ! .
$$

Note that in (4.2) the estimates are the same as in (4.1) but only for $k>\eta$. This suggests that for $\eta<0$, we have $\mathrm{B}_{\beta}(S)=\mathrm{A}_{\beta}(S)$, which will be proved below.

For generalization of the Taylor expansion in the critical case, we develop the Mellindomain analogue of an idea from [4], based on the splitting of $u^{M}$ provided by the decomposition

$$
U(\lambda)=\left(\mathbb{I}-\mathfrak{P}^{M}\right) U(\lambda)+\mathfrak{P}^{M} U(\lambda) .
$$

The first part is the Mellin transform of a function in $\mathrm{K}_{\beta}^{m}(K)$ and the second one has essentially a one dimensional structure - that is, the most important features of its structure are described by the behavior of functions of one variable - and it can be regularized in such a way that it splits again into two parts, one in the analytic class $\mathrm{B}_{\beta}(K)$, and the remaining part in $\mathrm{K}_{\beta}^{m}(K)$.

4.2. Mellin regularizing operator in one dimension. The main tool of the following analysis is a one-dimensional Mellin convolution operator:

Definition 4.2. We denote by $\mathfrak{K}: v \mapsto \mathfrak{K} v$ be the Mellin convolution operator defined by

$$
\mathscr{M}[\mathfrak{K} v](\lambda)=\mathrm{e}^{\lambda^{2}} \mathscr{M}[v](\lambda) .
$$

Owing to the strong decay properties of the kernel $\mathrm{e}^{\lambda^{2}}$ in the imaginary direction, the operator $\mathfrak{K}$ has analytic regularizing properties in the scales $\mathrm{K}_{\beta}^{m}$ and $\mathrm{J}_{\beta}^{m}$.

Proposition 4.3. Let $\beta \in \mathbb{R}$ and $m \geqslant 1$. There holds:

a) If $v \in \mathrm{K}_{\beta}^{m}\left(\mathbb{R}_{+}\right)$, then $\mathfrak{K} v \in \mathrm{A}_{\beta}\left(\mathbb{R}_{+}\right)$.

b) If $v \in \mathrm{J}_{-\frac{1}{2}}^{m}\left(\mathbb{R}_{+}\right)$with support in $I:=[0,1]$, then $\left.\mathfrak{K} v\right|_{I}$ belongs to the analytic class $\mathrm{B}_{-\frac{1}{2}}(I)$, and $v-\mathfrak{K} v \in \mathrm{K}_{-\frac{1}{2}}^{m}\left(\mathbb{R}_{+}\right)$.

c) If $v \in \mathrm{J}_{\beta}^{m}\left(\mathbb{R}_{+}\right)$with support in $I:=[0,1]$, and if $\beta<-\frac{1}{2}$ so that $v$ is continuous in 0 , then $\mathfrak{K} v$ is continuous in 0 as well, and $\mathfrak{K} v(0)=v(0)$.

The proof of this proposition is based on the following characterization of analytic classes by Mellin transformation:

Lemma 4.4. Let $\beta \in \mathbb{R}$ and $\eta=-\beta-\frac{1}{2}$. We set $I=(0,1)$. There holds 
MELLIN ANALYSIS OF WEIGHTED SOBOLEV SPACES WITH NONHOMOGENEOUS NORMS ON CONES3

a) Let $v \in \mathrm{K}_{\beta}^{1}\left(\mathbb{R}_{+}\right)$. Then $v$ belongs to $\mathrm{A}_{\beta}\left(\mathbb{R}_{+}\right)$if and only if $V:=\mathscr{M}[v]$ satisfies

$$
\exists C>0, \forall k \geqslant 1, \quad\left\{\int_{\operatorname{Re} \lambda=\eta}|\lambda|^{2 k}|V(\lambda)|^{2} \mathrm{~d} \operatorname{Im} \lambda\right\}^{\frac{1}{2}} \leqslant C^{k+1} k !
$$

b) Let $v \in \mathrm{J}_{-\frac{1}{2}}^{1}\left(\mathbb{R}_{+}\right)$. Then $\left.v\right|_{I}$ belongs to $\mathrm{B}_{-\frac{1}{2}}(I)$ if (4.4) is satisfied with $\eta=0$ and $V(\lambda):=\lambda^{-1} \mathscr{M}\left[r \partial_{r} v\right](\lambda)$.

Proof. a) According to Definition 4.1, $v \in \mathrm{A}_{\beta}\left(\mathbb{R}_{+}\right)$if and only if

$$
\exists C>1, \forall k \geqslant 0, \quad\left\|r^{\beta+k} \partial_{r}^{k} v\right\|_{0 ; \mathbb{R}_{+}} \leqslant C^{k+1} k !
$$

Using (3.18), one can see that this is equivalent to

$$
\exists C>1, \forall k \geqslant 0, \quad\left\|r^{\beta}\left(r \partial_{r}\right)^{k} v\right\|_{0 ; \mathbb{R}_{+}} \leqslant C^{k+1} k !
$$

Then a) is a consequence of the Parseval equality.

b) Let $v \in J_{-\frac{1}{2}}^{1}\left(\mathbb{R}_{+}\right)$. With $V(\lambda)=\lambda^{-1} \mathscr{M}\left[r \partial_{r} v\right](\lambda)$, for any $k \geqslant 1$ the function $\lambda^{k} V(\lambda)$ is the Mellin transform of $\left(r \partial_{r}\right)^{k} v$ on the line $\operatorname{Re} \lambda=0$. Thus, (4.4) with $\eta=0$ implies the analytic estimates

$$
\exists C>0, \forall k \geqslant 1, \quad\left\|r^{-\frac{1}{2}}\left(r \partial_{r}\right)^{k} v\right\|_{0 ; \mathbb{R}_{+}} \leqslant C^{k+1} k !
$$

Restricting this to $I$, and using Definition 4.1, we find that $\left.v\right|_{I} \in \mathrm{B}_{-\frac{1}{2}}(I)$.

Proof of Proposition 4.3. a) Let $v \in \mathrm{K}_{\beta}^{m}\left(\mathbb{R}_{+}\right)$and let $V$ be the Mellin transform of $v$. It is defined for $\operatorname{Re} \lambda=\eta$ and, in particular, the norm

$$
N_{0}:=\left\{\int_{\operatorname{Re} \lambda=\eta}|V(\lambda)|^{2} \mathrm{~d} \operatorname{Im} \lambda\right\}^{\frac{1}{2}}
$$

is finite. The Mellin transform of $\mathfrak{K} v$ is $\lambda \mapsto \mathrm{e}^{\lambda^{2}} V(\lambda)$. We have for any $k \geqslant 1$

$$
\begin{aligned}
\left\{\int_{\operatorname{Re} \lambda=\eta}|\lambda|^{2 k}\left|\mathrm{e}^{\lambda^{2}} V(\lambda)\right|^{2} \mathrm{~d} \operatorname{Im} \lambda\right\}^{\frac{1}{2}} & \leqslant N_{0} \sup _{\operatorname{Re} \lambda=\eta}|\lambda|^{k}\left|\mathrm{e}^{\lambda^{2}}\right| \\
& \leqslant C(\eta) N_{0} \sup _{\xi \geqslant 0} \xi^{k} \mathrm{e}^{-\xi^{2}}=C(\eta) N_{0}\left(\frac{k}{2 \mathrm{e}}\right)^{\frac{k}{2}} .
\end{aligned}
$$

Therefore condition (4.4) is satisfied for the Mellin transform of $\mathfrak{K} v$. By Lemma 4.4 a), $\mathfrak{K} v$ belongs to $A_{\beta}\left(\mathbb{R}_{+}\right)$.

b) Let $v \in J_{-\frac{1}{2}}^{m}\left(\mathbb{R}_{+}\right)$with support in $I$. By Corollary 3.19, $v \in J_{-\frac{1}{2}}^{1}\left(\mathbb{R}_{+}\right)$. Now, $V$ is defined as the Mellin transform of $r \partial_{r} v$ divided by $\lambda$. Thus $V$ coincides with $\mathscr{M}[v]$ where $\mathscr{M}[v]$ is well defined, and the Mellin transform of $\mathfrak{K} v$ is given by $\mathrm{e}^{\lambda^{2}} V(\lambda)$. With the same arguments as above, we prove that $\mathfrak{K} v$ satisfies the assumptions of Lemma $4.4 \mathrm{~b}$ ), hence $\left.\mathfrak{K} v\right|_{I} \in \mathrm{B}_{-\frac{1}{2}}(I)$. 
The Mellin transform of $v-\mathfrak{K} v$ is $\left(1-\mathrm{e}^{\lambda^{2}}\right) V(\lambda)$. Since $r^{-\frac{1}{2}}\left(r \partial_{r}\right)^{k} v \in \mathrm{L}^{2}\left(\mathbb{R}_{+}\right)$for $k=1, \ldots, m$ we have

$$
\sum_{k=1}^{m}\left\{\int_{\operatorname{Re} \lambda=0}|\lambda|^{2 k}|V(\lambda)|^{2} \mathrm{~d} \operatorname{Im} \lambda\right\}^{\frac{1}{2}}<\infty .
$$

The function $\lambda \mapsto\left(1-\mathrm{e}^{\lambda^{2}}\right)$ is bounded on the line $\operatorname{Re} \lambda=0$ and has a double zero at $\lambda=0$. Hence we deduce from (1) that

$$
\sum_{k=0}^{m}\left\{\int_{\operatorname{Re} \lambda=0}|\lambda|^{2 k}\left|\left(1-\mathrm{e}^{\lambda^{2}}\right) V(\lambda)\right|^{2} \mathrm{~d} \operatorname{Im} \lambda\right\}^{\frac{1}{2}}<\infty .
$$

Therefore $v-\mathfrak{K} v \in \mathrm{K}_{-\frac{1}{2}}^{m}\left(\mathbb{R}_{+}\right)$.

c) Let $v \in \mathrm{J}_{\beta}^{m}\left(\mathbb{R}_{+}\right)$with support in $I, \beta<-\frac{1}{2}$. It is sufficient to consider the case $m=1$ and $-\frac{3}{2}<\beta<-\frac{1}{2}$. With $\eta=-\beta-\frac{1}{2}$ we then have $0<\eta<1$. Let $V$ be the Mellin transform of $v$, and set $w=v-\mathfrak{K} v$. As above we have

$$
\mathscr{M}[w](\lambda)=\left(1-\mathrm{e}^{\lambda^{2}}\right) V(\lambda)=\frac{1-\mathrm{e}^{\lambda^{2}}}{\lambda} U(\lambda), \quad \text { where } U=\mathscr{M}\left[r \partial_{r} v\right] .
$$

Since the function $u=r \partial_{r} v$ belongs to $\mathrm{K}_{\beta}^{0}\left(\mathbb{R}^{+}\right)$and has support in $I, U$ is holomorphic for $\operatorname{Re} \lambda<\eta$, and $\mathscr{M}[w](\lambda)$ has the same property. It follows that $w \in \mathrm{K}_{\beta}^{1}\left(\mathbb{R}^{+}\right)$, which implies that $w$ is continuous at 0 and $w(0)=0$.

4.3. Generalized Taylor expansions. We are now ready for the definition of the splitting which replaces the Taylor expansion in the critical case: Let us assume that the natural number $M$ is critical. We are going to replace the homogeneous part

$$
\mathfrak{T}^{M} u=\sum_{|\alpha|=M} \partial_{\mathbf{x}}^{\alpha} u(\mathbf{0}) \frac{\mathbf{x}^{\alpha}}{\alpha !}
$$

of the corner Taylor expansion with a new operator $u \mapsto \mathfrak{K}^{M} u$ for which the point traces $\partial_{\mathbf{x}}^{\alpha} u(\mathbf{0})$ are replaced by moments defined thanks to the the dual basis (3.21) $\left(\varphi_{\gamma}^{M}\right)_{|\gamma|=M}$. Let us recall that:

$$
\int_{G} \frac{\vartheta^{\alpha}}{\alpha !} \varphi_{\gamma}^{M}(\vartheta) \mathrm{d} \vartheta=\delta_{\alpha \gamma}, \quad|\alpha|=|\gamma|=M, \quad \vartheta^{\alpha}=r^{-M} \mathbf{x}^{\alpha},
$$

and this dual basis served to define the projection operator $\mathfrak{P}^{M}: \mathrm{L}^{2}(G) \rightarrow \mathrm{P}^{M}(G)$ as

$$
\mathfrak{P}^{M} U=\sum_{|\alpha|=k}\left\langle U, \varphi_{\alpha}^{M}\right\rangle_{G} \frac{\vartheta^{\alpha}}{\alpha !} .
$$

Definition 4.5. Let $M \in \mathbb{N}$. For $u \in \mathscr{C}^{\infty}(\bar{K})$, let $\mathfrak{T}^{M-1} u$ be its Taylor expansion at 0 of order $M-1$, and $u^{M}=u-\mathfrak{T}^{M-1} u$ its Taylor remainder of order $M$, considered in polar coordinates $(r, \vartheta)$. With the dual basis (4.5), we define the moments of $u^{M}$ :

$$
\forall \alpha,|\alpha|=M, \quad d_{\alpha}(r)=\left\langle r^{-M} u^{M}(r, \cdot), \varphi_{\alpha}^{M}\right\rangle_{G}, \quad r>0 .
$$


MELLIN ANALYSIS OF WEIGHTED SOBOLEV SPACES WITH NONHOMOGENEOUS NORMS ON CONE\$5

Let us fix a cut-off function $\chi \in \mathscr{C}_{0}^{\infty}((-1,1)), \chi \equiv 1$ on $\left[-\frac{1}{2}, \frac{1}{2}\right]$. Then, using (4.3), the regularizing operator $\mathfrak{K}^{M} u$ is defined by

$$
\mathfrak{K}^{M} u=\sum_{|\alpha|=M} \mathfrak{K}\left(\chi d_{\alpha}\right) \frac{\mathbf{x}^{\alpha}}{\alpha !} .
$$

Remark 4.6. For $M=0, d_{\alpha} \equiv d_{0}$ is the mean value of $u(r, \cdot)$ over $G$ and $\mathfrak{K}^{0} u=\mathfrak{K}\left(\chi d_{0}\right)$. In particular, if $u$ is continuous in $\mathbf{0}$, then both $d_{0}$ and $\mathfrak{K}\left(\chi d_{0}\right)$ are continuous in 0 , and $\mathfrak{K}^{0} u(\mathbf{0})=u(\mathbf{0})$, see Proposition $\left.4.3 \mathrm{c}\right)$. More generally, for sufficiently smooth $u$, one has

$$
d_{\alpha}(0)=\mathfrak{K}\left(\chi d_{\alpha}\right)(0)=\partial_{\mathbf{x}}^{\alpha} u(\mathbf{0}) .
$$

The moments $d_{\alpha}$ are well-defined in the critical case and have the following properties:

Proposition 4.7. Let $\beta$ be real such that $-\beta-\frac{n}{2}$ coincides with a non-negative integer $M$. For $m>M$, let $u \in \mathrm{J}_{\beta}^{m}(K)$ with support in $B(0,1)$. Then the moments $d_{\alpha}$ as defined in (4.7) satisfy:

a) For all $|\alpha|=M, \chi d_{\alpha} \in \mathrm{J}_{-\frac{1}{2}}^{m}\left(\mathbb{R}_{+}\right)$.

b) If $\forall|\alpha|=M, \chi d_{\alpha} \in \mathrm{K}_{-\frac{1}{2}}^{m}\left(\mathbb{R}_{+}\right)$, then $u-\mathfrak{T}^{M-1} u$ belongs to $\mathrm{K}_{\beta}^{m}(K)$.

Proof. We can assume without restriction that $\chi u=u$. Let us set $v=u-\chi \mathfrak{T}^{M-1} u$. Then $\chi d_{\alpha}[u]=d_{\alpha}[v]$ and

$$
v-\mathfrak{T}^{M-1} v=v \quad \text { and } \quad u-\mathfrak{T}^{M-1} u=v-(1-\chi) \mathfrak{T}^{M-1} u .
$$

Since $(1-\chi) \mathfrak{T}^{M-1} u$ belongs to $\mathrm{K}_{\beta}^{m}(K)$, we can replace $u$ with $v$ and omit the cut-off $\chi$. We still denote $v$ by $u$. The Mellin transform $U(\lambda)$ of $u$ is holomorphic in the half-plane $\operatorname{Re} \lambda<M$ and, by Theorem 3.22, the norm

$$
\begin{aligned}
\sup _{b \in\left(M-\frac{1}{2}, M\right)}\left\{\int_{\substack{|\operatorname{Im} \lambda| \leqslant 1 \\
\operatorname{Re} \lambda=b}}\left\|\left(\mathbb{I}-\mathfrak{P}^{M}\right) U(\lambda)\right\|_{m ; G}^{2}+\mid \lambda\right. & -\left.M\right|^{2}\left\|\mathfrak{P}^{M} U(\lambda)\right\|_{m ; G}^{2} \mathrm{~d} \operatorname{Im} \lambda \\
& \left.+\int_{\substack{|\operatorname{Im} \lambda| \geqslant 1 \\
\operatorname{Re} \lambda=b}}\|U(\lambda)\|_{m ; G ; \lambda}^{2} \mathrm{~d} \operatorname{Im} \lambda\right\}^{\frac{1}{2}}
\end{aligned}
$$

is bounded by $C\|u\|_{J_{\beta}^{m}(K)}$.

As a mere consequence of the definition of $\mathfrak{P}^{M}$, see (4.6), we have the uniform inequality for $\operatorname{Re} \lambda<M$

$$
\left\|\mathfrak{P}^{M} U(\lambda)\right\|_{m ; G ; \lambda} \leqslant C\|U(\lambda)\|_{m ; G ; \lambda} .
$$

Hence we deduce from estimates (4.9) that

$$
\sup _{b \in\left(M-\frac{1}{2}, M\right)} \int_{\operatorname{Re} \lambda=b}\left\|\left(\mathbb{I}-\mathfrak{P}^{M}\right) U(\lambda)\right\|_{m ; G ; \lambda}^{2} \mathrm{~d} \operatorname{Im} \lambda \leqslant C\|u\|_{\mathrm{J}^{m}(K)}^{2} .
$$

Thus Theorem 3.2 yields that $\mathscr{M}^{-1}\left[\left(\mathbb{I}-\mathfrak{P}^{M}\right) U\right]$ belongs to $\mathrm{K}_{\beta}^{m}(K)$. 
Let us set $D_{\alpha}=\mathscr{M}\left[d_{\alpha}\right]$. We have

$$
\begin{aligned}
D_{\alpha}(\lambda) & =\mathscr{M}\left[\left\langle r^{-M} u^{M}, \varphi_{\alpha}^{M}\right\rangle_{G}\right](\lambda) \\
& =\mathscr{M}\left[\left\langle u^{M}, \varphi_{\alpha}^{M}\right\rangle_{G}\right](\lambda+M) \\
& =\left\langle U(\lambda+M), \varphi_{\alpha}^{M}\right\rangle_{G} .
\end{aligned}
$$

Therefore, by formulas (4.5) and (4.6), we find

$$
D_{\alpha}(\lambda)=\left\langle\mathfrak{P}^{M} U(\lambda+M), \varphi_{\alpha}^{M}\right\rangle_{G} .
$$

a) We deduce from (1) and (4.9) that $D_{\alpha}$ is holomorphic in the half-plane $\operatorname{Re} \lambda<0$ and that

$$
\sup _{b \in\left(-\frac{1}{2}, 0\right)}\left\{\int_{\operatorname{Re} \lambda=b}\left(|\lambda|^{2}+|\lambda|^{2 m}\right)\left|D_{\alpha}(\lambda)\right|^{2} \mathrm{~d} \operatorname{Im} \lambda\right\}^{\frac{1}{2}}
$$

is bounded. This allows to prove that $d_{\alpha} \in \mathrm{J}_{-\frac{1}{2}}^{m}\left(\mathbb{R}_{+}\right)$.

b) If $d_{\alpha} \in \mathrm{K}_{-\frac{1}{2}}^{m}\left(\mathbb{R}_{+}\right)$, then

$$
\int_{\operatorname{Re} \lambda=0}\left(1+|\lambda|^{2 m}\right)\left|D_{\alpha}(\lambda)\right|^{2} \mathrm{~d} \operatorname{Im} \lambda
$$

is bounded. Since by (1) and (4.5):

$$
\mathfrak{P}^{M} U(\lambda+M)=\sum_{|\alpha|=M} D_{\alpha}(\lambda) \frac{\vartheta^{\alpha}}{\alpha !}
$$

we find

$$
\int_{\operatorname{Re} \lambda=M}\left\|\mathfrak{P}^{M} U(\lambda)\right\|_{m ; G ; \lambda}^{2} \mathrm{~d} \operatorname{Im} \lambda<\infty
$$

hence $\mathscr{M}^{-1}\left[\mathfrak{P}^{M} U\right] \in \mathrm{K}_{\beta}^{m}(K)$. Since $\mathscr{M}^{-1}\left[\left(\mathbb{I}-\mathfrak{P}^{M}\right) U\right] \in \mathrm{K}_{\beta}^{m}(K)$, this ends the proof.

We conclude this section with a result about the generalized Taylor expansion at the corner in the critical case. The homogeneous part of critical degree $\sum_{|\alpha|=M} \partial_{\mathbf{x}}^{\alpha} u(\mathbf{0}) \frac{\mathbf{x}^{\alpha}}{\alpha !}$ does not make sense, because the Taylor coefficients $\partial_{\mathbf{x}}^{\alpha} u(\mathbf{0})$ are not bounded with respect to the $\mathrm{J}_{\beta}^{m}$ norm in this case. But one can replace the constants $\partial_{\mathbf{x}}^{\alpha} u(\mathbf{0})$ by "generalized constants", namely the analytic functions $\mathfrak{K}\left(\chi d_{\alpha}\right)$, which means that the homogeneous part of degree $M$ of the Taylor expansion is replaced by $\mathfrak{K}^{M} u$, which is not polynomial but belongs to the analytic class $\mathrm{B}_{\beta}(S)$. The "Taylor remainder" then belongs to $\mathrm{K}_{\beta}^{m}(K)$.

Theorem 4.8. Let $\beta$ be such that $-\beta-\frac{n}{2}=M \in \mathbb{N}$ and let $u \in J_{\beta}^{m}(K)$ with support in $B(\mathbf{0}, 1)$. Then

$$
u-\mathfrak{T}^{M-1} u-\mathfrak{K}^{M} u \in \mathrm{K}_{\beta}^{m}(S) \text { and } \mathfrak{K}^{M} u \in \mathrm{B}_{\beta}(S) .
$$

Proof. Let $u \in J_{\beta}^{m}(K)$ with support in $B(\mathbf{0}, 1)$. By Proposition 4.7 a), for all $|\alpha|=M, \chi d_{\alpha}$ belongs to $J_{-\frac{1}{2}}^{m}\left(\mathbb{R}_{+}\right)$. By Proposition $\left.4.3 \mathrm{~b}\right)$, we deduce that $\mathfrak{K}\left(\chi d_{\alpha}\right)$ belongs to $\mathrm{B}_{-\frac{1}{2}}(I)$. Let us consider the function

$$
v_{\alpha}: S \ni \mathbf{x} \mapsto \mathfrak{K}\left(\chi d_{\alpha}\right)(r)
$$


and the class $\mathrm{B}_{-\frac{n}{2}}(S)$. This class is associated with $\eta=0$. Therefore, using Lemma 3.21 we deduce that $v_{\alpha} \in \mathrm{B}_{-\frac{n}{2}}(S)$ as a direct consequence of the fact that $\mathfrak{K}\left(\chi d_{\alpha}\right) \in \mathrm{B}_{-\frac{1}{2}}(I)$. Multiplying by $\mathbf{x}^{\alpha}$, we find that $\mathbf{x} \mapsto \mathbf{x}^{\alpha} v_{\alpha}(\mathbf{x})$ belongs to $\mathrm{B}_{-\frac{n}{2}-M}(S)=\mathrm{B}_{\beta}(S)$. Finally $\mathfrak{K}^{M} u$ belongs to $\mathrm{B}_{\beta}(S)$.

Let $v=u-\mathfrak{T}^{M-1} u-\mathfrak{K}^{M} u$. It remains to show that $v \in \mathrm{K}_{\beta}^{m}(S)$. Denote by $d_{\alpha}[v]$ the moments of $v$ defined like in (4.7). We notice that

$$
\chi d_{\alpha}[v]=\chi d_{\alpha}-\chi \mathfrak{K}\left(\chi d_{\alpha}\right) .
$$

But Proposition 4.7 a) yields $\chi d_{\alpha} \in J_{-\frac{1}{2}}^{m}\left(\mathbb{R}_{+}\right)$and then by Proposition $4.3 \mathrm{~b}$ ) we get $\chi d_{\alpha}-$ $\mathfrak{K}\left(\chi d_{\alpha}\right) \in \mathrm{K}_{-\frac{1}{2}}^{m}\left(\mathbb{R}_{+}\right)$, hence $\chi d_{\alpha}[v] \in \mathrm{K}_{-\frac{1}{2}}^{m}\left(\mathbb{R}_{+}\right)$. The regularity $v \in \mathrm{K}_{\beta}^{m}(S)$ is then a consequence of Proposition $4.7 \mathrm{~b}$ ).

Corollary 4.9. Let $\beta$ be such that $-\beta-\frac{n}{2}=M \in \mathbb{N}$ and $m>M$. Then the space $\mathrm{K}_{\beta}^{m}(S)$ is not closed in $\mathrm{J}_{\beta}^{m}(S)$ and the quotient $\mathrm{J}_{\beta}^{m}(S) / \mathrm{K}_{\beta}^{m}(S)$ is infinite dimensional.

\section{CONCLUSION}

Theorems 3.22 and 4.8 can advantageously be used for the analysis of second order elliptic boundary value problems in domains $\Omega$ with corners: Let $L$ be the interior operator and $B$ the operator on the boundary. $L$ is supposed to be elliptic on $\bar{\Omega}$ and $B$ to cover $L$ on $\partial \Omega$. The order $d$ of $B$ is 0 or 1 .

Theorem 3.22 fully characterizes the spaces $J_{\beta}^{m}$ by Mellin transformation. This is an essential tool for stating necessary and sufficient conditions for $(L, B)$ to define a Fredholm operator:

$$
\mathrm{J}_{\beta}^{m}(\Omega) \longrightarrow \mathrm{J}_{\beta+2}^{m-2}(\Omega) \times \Gamma_{\partial \Omega} \mathrm{J}_{\beta+d}^{m-d}(\Omega)
$$

where $\Gamma_{\partial \Omega}$ denotes the trace operator on $\partial \Omega$. When $\mathrm{K}_{\beta}^{m}$ spaces are involved instead, this condition is the absence of poles for the corner Mellin resolvents on certain lines $\{\operatorname{Re} \lambda=$ const\}, see [3]. Theorem 3.22 allows to prove by Mellin transformation that the necessary and sufficient condition associated with spaces $\mathrm{J}_{\beta}^{m}$ is the injectivity modulo polynomials (cf. $[2,1]$ ) on similar lines in the complex plane.

Theorem 4.8 allows to prove an analytic shift theorem in $\mathrm{J}_{\beta}^{m}$ spaces for elliptic $(L, B)$ with analytic coefficients: Roughly, this means that if a solution $u$ belongs to $\mathrm{J}_{\beta}^{2}(\Omega)$ and is associated with a right hand side in $\operatorname{RB}_{\beta}(\Omega):=\mathrm{B}_{\beta+2}(\Omega) \times \Gamma_{\partial \Omega} \mathrm{B}_{\beta+d}(\Omega)$, then $u$ belongs to $\mathrm{B}_{\beta}(\Omega)$. This result relies on

(1) The analytic shift theorem in the scale $\mathrm{K}_{\beta}^{m}$ : If $u \in \mathrm{K}_{\beta}^{2}(\Omega)$ and the right hand sides belongs to $\operatorname{RA}_{\beta}(\Omega)$, then $u \in \mathrm{A}_{\beta}(\Omega)$.

(2) The splitting (4.10).

The analytic shift theorem in the scale $\mathrm{K}_{\beta}^{m}$, that is with homogeneous norms, can be proved by a "standard" technique of dyadic refined partitions towards the corners combined with local analytic estimates in smooth regions. This technique cannot be directly applied to spaces with non-homogeneous norms, hence the utility of the splitting (4.10).

See [1, Part II] for details. 


\section{REFERENCES}

[1] M. Costabel, M. Dauge, S. Nicaise. Corner Singularities and Analytic Regularity for Linear Elliptic Systems In preparation.

[2] M. Dauge. Elliptic Boundary Value Problems in Corner Domains - Smoothness and Asymptotics of Solutions. Lecture Notes in Mathematics, Vol. 1341. Springer-Verlag, Berlin 1988.

[3] V. A. Kondrat'Ev. Boundary-value problems for elliptic equations in domains with conical or angular points. Trans. Moscow Math. Soc. 16 (1967) 227-313.

[4] V. A. Kozlov, V. G. MaZ'YA, J. Rossmann. Elliptic boundary value problems in domains with point singularities. Mathematical Surveys and Monographs, 52. American Mathematical Society, Providence, RI 1997.

[5] V. A. Kozlov, V. G. Maz'Ya, J. Rossmann. Spectral Problems Associated with Corner Singularities of Solutions to Elliptic Equations. Mathematical Surveys and Monographs, 85. American Mathematical Society, Providence, RI 2001.

[6] V. G. MaZ'Ya, B. A. Plamenevskit. Weighted spaces with nonhomogeneous norms and boundary value problems in domains with conical points. Amer. Math. Soc. Transl. (2) 123 (1984) 89-107.

[7] S. A. NAZAROV. Vishik-Lyusternik method for elliptic boundary value problems in regions with conical points. I. The problem in a cone. Siberian Math. J. 22 (1981) 594-611.

[8] S. A. Nazarov, B. A. Plamenevskil. The Neumann problem for selfadjoint elliptic systems in a domain with a piecewise-smooth boundary. Amer. Math. Soc. Transl. (2) 155 (1993) 169-206.

[9] S. A. Nazarov, B. A. Plamenevs KiI. Elliptic Problems in Domains with Piecewise Smooth Boundaries. Expositions in Mathematics 13. Walter de Gruyter, Berlin 1994.

\section{ADDRESSES}

IRMaR, Université de Rennes 1, Campus de Beaulieu, 35042 Rennes Cedex, France

E-mail address: martin.costabel@univ-rennesl.fr

IRMaR, Université de Rennes 1, Campus de Beaulieu, 35042 Rennes Cedex, France

E-mail address: monique. dauge@univ-rennes1. fr

LAMAV, FR CNRS 2956, Université LiLle Nord De France, UVHC, 59313 VALEnciennes CEDEX 9, FRANCE.

E-mail address: snicaise@univ-valenciennes.fr 